\title{
STRESS ANALYSIS OF COMPOSITE CYLINDRICAL SHELLS WITH AN ELLIPTICAL CUTOUT
}

\author{
E. Oterkus, ${ }^{\dagger}$ E. Madenci ${ }^{\star}$ \\ The University of Arizona, Tucson, AZ 85721 \\ M. P. Nemeth ${ }^{\S}$ \\ NASA Langley Research Center, Hampton, VA 23681
}

\begin{abstract}
A special-purpose, semi-analytical solution method for determining the stress and deformation fields in a thin laminated-composite cylindrical shell with an elliptical cutout is presented. The analysis includes the effects of cutout size, shape, and orientation; nonuniform wall thickness; oval-cross-section eccentricity; and loading conditions. The loading conditions include uniform tension, uniform torsion, and pure bending. The analysis approach is based on the principle of stationary potential energy and uses Lagrange multipliers to relax the kinematic admissibility requirements on the displacement representations through the use of idealized elastic edge restraints. Specifying appropriate stiffness values for the elastic extensional and rotational edge restraints (springs) allows the imposition of the kinematic boundary conditions in an indirect manner, which enables the use of a broader set of functions for representing the displacement fields. Selected results of parametric studies are presented for several geometric parameters that demonstrate that analysis approach is a powerful means for developing design criteria for laminatedcomposite shells.
\end{abstract}

\section{Introduction}

Cutouts in cylindrical shell-type components are unavoidable in the construction of aerospace structures. This fact is significant because the structural failure of these components usually begins near the cutout because of high stress concentrations that initiate the formation of cracks. Hence, a cutout can trigger a local failure at a load level lower than the global failure load of a corresponding shell without a cutout. As a result, preliminary-design sizing of a cylindrical shell with a cutout is often based on the magnitude of the stress

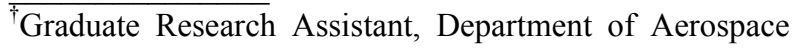
and Mechanical Engineering.

$\$$ Professor, Department of Aerospace and Mechanical Engineering, Member AIAA.

$\S$ Senior Research Engineer, Mechanics of Structures and Materials Branch, Associate Fellow AIAA

Copyright (C) 2005 by the American Institute of Aeronautics and Astronautics. All rights reserved.
}

concentrations near the cutout. Therefore, an accurate assessment of the stress concentrations in a given shell subjected to various types of loading and support conditions is essential to the development of safe and reliable designs. Moreover, validated special-purpose analysis tools that enable rapid parametric studies would be very valuable to structural designers and for the development of new design criteria and design concepts.

Several analytical, numerical and experimental studies have been conducted during the past sixty years to determine stress distributions in cylindrical shells with a cutout and subjected to various types of loadings; such as, axial tension and compression, torsion, and internal and external pressure. Pioneering analytical work was conducted by Lurie ${ }^{1,2}$ to investigate the effect of axial tension and internal pressure, and shell curvature, on the stress concentrations around a circular cutout in the 1940s. Many years later, analytical studies were presented by Lekkerkerker, ${ }^{3}$ Van Dyke, ${ }^{4}$ Ashmarin, ${ }^{5}$ Murthy et al., ${ }^{6}$ Guz et al., ${ }^{7}$ and Van Tooren et al. ${ }^{8}$ that further investigated the effects of various factors on the stress concentrations around a cutout in a cylindrical shell. Similarly, experimental investigations have been conducted by Tennyson, ${ }^{9}$ Starnes, ${ }^{10}$ Pierce and Chou, ${ }^{11}$ Bull, ${ }^{12}$ and Zirka and Chernopiskii, ${ }^{13}$ and numerical studies have been conducted by Liang et al., ${ }^{14}$ and Shnerenko and Godzula. ${ }^{15}$ In 1964 and 1972, respectively, Hicks ${ }^{16}$ and Ebner and Jung ${ }^{17}$ summarized the results obtained from several of these previous studies and provided extensive lists of references related to this problem. Most of these previous studies are for isotropic cylindrical shells with a circular cutout. Only a few of these studies, such as those presented by Pierce and $\mathrm{Chou}^{11}$ and by Murthy et al., ${ }^{6}$ address the effects of cutout shape (elliptical cutouts) on the stress concentrations.

Mitigation of high stress concentrations by tailoring shell-wall thickness, material orthotropy and anisotropy, and cutout reinforcement are also important considerations in the design of aerospace structures made of lightweight composite materials. Likewise, the potential for using shells of non-circular cross section are relevant to fuselage-like structures. However, only a few studies have considered these effects. For 
example, the influence of wall-thickness variation on the stresses in axially loaded composite cylindrical shells, without a cutout, has been investigated by Li et al. ${ }^{18}$ Although numerous analyses exist in the literature on the analysis of shells with circular cross sections, only a few include non-circular cross sections. Sheinman and Firer $^{19}$ provided an analytical investigation of stresses in laminated cylindrical shells with arbitrary non-circular cross sections. More recently, Hyer and Wolford ${ }^{20,21}$ studied the effect of non-circular cross sections on damage initiation and progressive failure in composite cylinders by employing the finite element method.

The objective of the present study is to present a special-purpose analysis for a laminated-composite cylindrical shell with an elliptical cutout that can be used to rapidly, and parametrically, investigate the effects of shell curvature; cutout size, shape, and orientation; and ply lay-up on stress-resultant concentrations near the cutout. The analysis is applicable to thin-walled cylindrical shells with nonuniform wall thickness, a non-circular (e.g., oval) cross section, and subjected to tension, torsion, and bending loads as illustrated in Fig. 1.

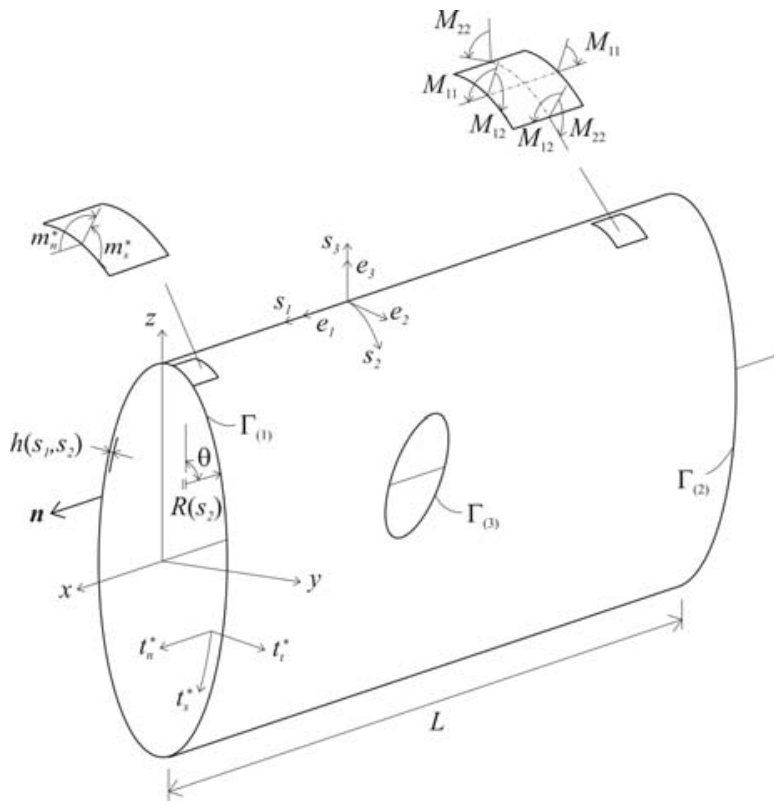

Fig. 1 Geometry, coordinates systems, and applied edge tractions for an oval cylindrical shell with an elliptical cutout and non-uniform wall thickness.

To accomplish this objective, an overview of the analysis is presented first. Next, the boundary value problem is defined along with the kinematics and stress-strain relations used in the analysis. Then, the derivation of the equations governing the response and numerical procedure are described. Finally, selected numerical results for oval and circular cylindrical shells with either circular or elliptical cutouts and subjected to either tension, torsion, or pure-bending loads are presented.

\section{Analysis Overview}

The analytical approach used herein permits the determination of the pointwise variation of displacement and stress components. It is based on the principle of stationary potential energy, but utilizes local and global functions that are not required to satisfy the kinematic boundary conditions directly. Thus, the choice of local and global functions is not limited by a particular type of kinematic boundary condition. The kinematic boundary conditions are imposed by employing the Lagrange multiplier method. Both local and global functions are used, in contrast to the traditional approach, to enhance the robustness of the analysis method. In particular, the local functions are used to capture rapidly varying stress and strain gradients and local deformations near a cutout. Toward that goal, Laurent series are used for the local functions and are expressed in terms of the mapping functions introduced by Lekhnitskii. ${ }^{22}$ Fourier series are used for the global functions and are used to capture the overall deformation and stress fields. The kinematic admissibility requirements on the local and global functions are relaxed by defining that the edges of the shell are supported by extensional and rotational springs. Zero-valued displacement and rotation kinematic boundary conditions are enforced in an indirect manner by specifiying values for the spring stiffnesses that are large compared to the corresponding shell stiffnesses. This approach effectively yields a prescribed kinematic boundary condition in the limit as the relative stiffness of the spring becomes much greater than the corresponding shell stiffness. Similarly, values for the spring stiffnesses can be selected that correspond to a given uniform elastic restraint along an edge, similar to that provided by an end-ring. This capability is important, and useful, because in some test fixtures or actual structures the edge supports may not be stiff enough to simulate a fully clamped boundary condition or flexible enough to simulate a simply supported boundary condition.

As suggested by $\mathrm{Li}$ et al. ${ }^{18}$ and Sheinman and Firer, ${ }^{19}$ nonuniform wall-thickness variations of a shell, which lead to non-uniform laminate stiffnesses, are represented by using trigonometric series. Specifically, nonuniform shell-wall thickness is represented in the present study by perturbing the ply thicknesses with a function that is periodic in either the longitudinal or the circumferential direction. The variation in wall thickness is accounted for by adjusting the lamina properties, resulting in nonhomogeneous in-plane and bending stiffness matrices. The nonuniform shell curvature associated with a noncircular cross section is 
represented by using trigonometric series for the coordinates of an oval-cross-section shell reference surface. $^{23}$ The aspect ratio, or out-of-roundness, of the cross-section is represented in the analysis by using an eccentricity parameter.

In the derivation of the equations governing the response, the total potential energy consists of the elastic strain energy of the shell, the elastic edge restraints and the potential energy of the applied loads. The conditions that may arise from the choice of displacement approximations without any kinematic restrictions are treated as constraint equations, and the potential energy arising from constraint reactions is invoked into the total potential energy through the use of Lagrange multipliers. The equations governing the shell response are obtained by enforcing the requirement that the first variation of the total potential energy vanish. The evaluation of the area integrals appearing in the potential energy are achieved numerically by using a basic quadrature method in conjunction with standard triangulation of the entire domain described by Shewchuk. ${ }^{24}$ Solution to the equations governing the response are obtained by using a standared Gaussian elimination procedure, which yields the generalized displacement coefficients and, thus, the stress and strain fields. The accuracy of the analysis depends on the number of terms used for the functional representation of the displacement fields. As the number of terms increases, the results converge to the exact solution.

\section{Representation of Shell Geometry}

The geometry of a thin-walled, noncircular, cylindrical shell of length $L$ and with an elliptical cutout located at the shell mid-length is shown in Fig. 1. The origin of the global Cartesian coordinate system, $(x, y, z)$ is located at an end point of the longitudinal axis of the shell. As shown in Fig. 1, the $x$-axis coincides with the longitudinal axis of the shell. The $y$ and $z$ coordinates span the cross-sectional plane. A curvilinear coordinate system is also attached to the mid-surface of the cylindrical shell. The coordinates of points in the longitudinal, circumferential (tangential), and normal-to-the-surface (transverse) directions of the shell are denoted by $\left(s_{1}, s_{2}, s_{3}\right)$, and the corresponding unit base vectors are $\left\{\mathbf{e}_{1}, \mathbf{e}_{2}, \mathbf{e}_{3}\right\}$.

Following Romano and Kempner, ${ }^{23}$ the non-circular cross-section of the cylindrical shell is defined as an oval with the coordinates, $y$ and $z$ expressed as

$$
y=R_{0} \sum_{m=1,3,5,7, \ldots} a_{m}(\xi) \sin \frac{m s_{2}}{R_{0}}
$$

and

$$
z=R_{0} \sum_{m=1,3,5,7, \ldots} b_{m}(\xi) \cos \frac{m s_{2}}{R_{0}}
$$

where $\xi$ represents the eccentricity of the oval cross section and $R_{0}$ is the equivalent radius of a circular cylindrical shell that has the same circumference as that of the oval cylindrical shell. The circumferential coordinate, $s_{2}$ varies between 0 and $2 \pi R_{0}$. The derivation of Eqs. (1) along with the explicit forms of the coefficients $a_{m}(\xi)$ and $b_{m}(\xi)$ are given by Madenci and Barut. ${ }^{25}$

As derived by Romano and Kempner, ${ }^{23}$ the coordinates $y$ and $z$ in Eqs. (1a) and (1b) can be related to the radius of curvature of an oval-crosssection cylindrical shell, $R\left(s_{2}, \xi\right)$, by

$$
R\left(s_{2}, \xi\right)=\frac{R_{0}}{1+\xi \cos \left(2 s_{2} / R_{0}\right)}
$$

Therefore, $\xi=0$ implies no eccentricity and corresponds to a circular cross section with radius $R_{0}$. For positive values of the eccentricity parameter, $\xi$, the $z$-coordinate becomes the major axis and the $y$ coordinate becomes the minor axis. For negative values of $\xi$, the major and minor axes switch to the $y$ and $z$-axes, respectively. The range of values of the eccentricity parameter, $\xi$, is bounded by $-1<\xi<1$.

As shown in Fig. 1, the cylindrical shell contains a cutout. The shape of this cutout is defined such that if the shell is cut along a generator and flattened into a plane, the cutout becomes an ellipse with major and minor axes denoted by $a$ and $b$, respectively. For simplicity and convenience, the cutout is referred to herein as an "elliptical" cutout. Because the domain of the analysis shown in Fig. 2 corresponds to a similar flat region, a subsequent mapping of the ellipse to a unit circle is possible, which enables the use of Laurent series expansions for the local functions. Note that the special case of a "circular" cutout is given by $a=b$.

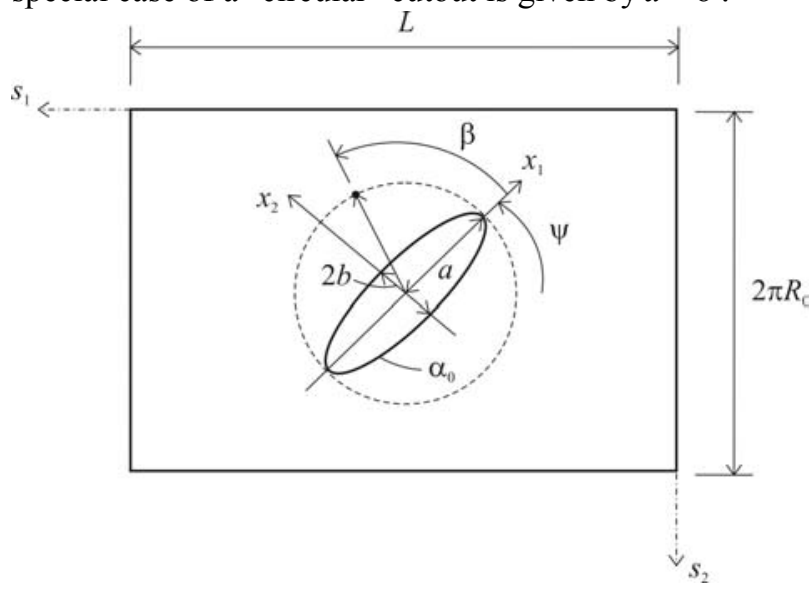

Fig. 2 Computational domain of a cylindrical shell with an elliptical cutout. 
In the flat analysis domain, the minor and major axes of the ellipse are aligned with a local coordinate system, $\left(x_{1}, x_{2}\right)$, whose origin is located at the center of the cutout and coincides with the origin of the parameter grid, given by constant values of $s_{1}$ and $s_{2}$, that forms the curvilinear coordinates $\left(s_{1}, s_{2}\right)$ on the cylindrical shell mid-surface. The orientation of the elliptical cutout is arbitrary with respect to the longitudinal shell axis. Hence, the orientation of the local $x_{1}$ - axis (major axis) of the cutout and the longitudinal $s_{1}$-axis of the cylindrical shell is denoted by the angle, $\psi$. The elliptical coordinates, $\alpha$ and $\beta$, representing a family of confocal ellipses and hyperbolas, respectively, are utilized in order to obtain the stress-resultant distribution in the direction tangent to the cutout boundary. The coordinate $\alpha$ is equal to $\alpha_{0}=\tanh ^{-1}(b / a)$ on the particular ellipse that corresponds to the elliptical cutout. The other coordinate, $\beta$, varying from 0 to $2 \pi$, is known as the eccentric angle and is related to the $\left(x_{1}, x_{2}\right)$ coordinate system by $x_{1}=a \cos \beta$ and $x_{2}=b \sin \beta$. The eccentric angle $\beta$ is similar to the angle used for polar coordinates.

The symmetrically laminated cylindrical shells considered herein are made of $K$ specially orthotropic layers, and each layer has an orientation angle, $\theta_{k}$, that is defined with respect to the $s_{1}$-axis. Each layer also has elastic moduli $E_{L}$ and $E_{T}$, shear modulus, $G_{L T}$ and Poisson's ratio $v_{L T}$, where the subscripts $L$ and $T$ represent the longitudinal (fiber) and transverse principal material directions, respectively.

As for the shell thickness variation, the non-uniform wall thickness of the shell is denoted by $h\left(s_{1}, s_{2}\right)$, and its variation is included by assuming that the thickness of each ply, $t_{k}$, varies as a function of the curvilinear coordinates in the form

$t_{k}\left(s_{1}, s_{2}\right)=t_{k 0}\left(1-\varepsilon_{1} \operatorname{Cos}\left(\frac{2 \pi m_{1} s_{1}}{L}\right)-\varepsilon_{2} \operatorname{Cos}\left(\frac{m_{2} s_{2}}{R_{0}}\right)\right)$

where $t_{k 0}$ denotes the nominal thickness of the $k^{\text {th }}$ layer in the laminate, and the parameters $\left(m_{1}, m_{2}\right)$ and $\left(\varepsilon_{1}, \varepsilon_{2}\right)$ respectively, denote the wave numbers and the amplitudes of the periodic thickness variation in the longitudinal and circumferential directions. While the wall thickness of the shell is allowed to vary across the shell surface, the aspect ratio of the plies through the thickness is maintained, thus making the thickness variation of each ply to remain conformable to each other throughout the shell surface. A periodic thickness variation in the longitudinal direction is obtained by setting $\varepsilon_{1} \neq 0$ and $\varepsilon_{2}=0$, and in the circumferential direction by $\varepsilon_{1}=0$ and $\varepsilon_{2} \neq 0$. A shell with uniform thickness, $t_{k}=t_{k 0}$, is obtained by setting $\varepsilon_{1}=0$ and $\varepsilon_{2}=0$.

\section{Boundary Conditions and External Loads}

To facilitate a general imposition of prescribed boundary tractions, displacements, or rotations; the external as well as the internal edge boundary $\Gamma$ of the shell is decomposed into

$\Gamma=\Gamma_{(1)}+\Gamma_{(2)}+\Gamma_{(3)}$

As shown in Fig. 1, $\Gamma_{(1)}$ and $\Gamma_{(2)}$ denote the external edge boundary of the cylindrical shell and $\Gamma_{(3)}$ represents the traction-free internal edge boundary around the cutout. The unit vector normal to an edge is represented byn. Throughout this paper, a variable with the superscript "*" is treated as a known quantity, arising from the externally applied loads or from prescribed displacements and rotations. Also, the subscripts $n, s$, and $t$ denote the directions normal, tangent, and transverse (through-the-thickness) to the boundary, respectively. The details of how prescribed edge loads and displacements are imposed in the analysis are presented subsequently.

\section{Prescribed edge loads}

External loads are applied to a shell by specifying values for the positive-valued stress resultants shown in Fig. 1. More precisely, the membrane loads applied to the $\ell^{\text {th }}$ boundary segment, $\Gamma_{(\ell)}$, are given by

$N_{11}=t_{n}^{*}$

$N_{12}=t_{s}^{*}$

where $\mathrm{N}_{11}$ and $\mathrm{N}_{12}$ are the axial and shear stress resultants, respectively, defined in the cylindrical coordinate system. Likewise, shell-wall bending loads that are applied to the $\ell^{\text {th }}$ boundary segment are given by

$$
\begin{aligned}
& M_{11}=-m_{n}^{*} \\
& M_{11,1}+2 M_{12,2}=t_{t}^{*}-2 m_{s, 2}^{*}
\end{aligned}
$$

where $M_{11}$ and $M_{12}$ are the pure-bending and twisting stress resultants, respectively, defined in the cylindrical coordinate system. Moreover, the left-hand side of Eq. (6b) is the Kirchhoff shear stress resultant of classical shell theory.

As a matter of convenience, the analysis is formulated to also permit the specification of concentrated forces and moments that are transmitted to the ends of the shell as if through a rigid end-ring, as shown in Fig. 3. Presently, the concentrated force $P_{n}^{*}$ and the concentrated axial torque $P_{s}^{*}$ are included in the 
analysis. The force $P_{n}^{*}$ is simulated in the analysis by specifiying a uniform distribution of the axial displacement, with the unknown magnitude $\Delta_{n}$, such that

$\int_{\Gamma_{(\ell)}} N_{11} d \Gamma=P_{n}^{*}$

Likewise, the torque $P_{s}^{*}$ is simulated by specifiying a uniform distribution of the tangential displacement, with the unknown magnitude $\Delta_{s}$, such that

$$
\int_{\Gamma_{(\ell)}} N_{12} d \Gamma=P_{s}^{*}
$$

The analytical process that is used to ensure that the magnitudes of $\Delta_{n}$ and $\Delta_{s}$ correspond to the specified values of $P_{n}^{*}$ and $P_{s}^{*}$, respectively, is described in the following section and in Appendix A.

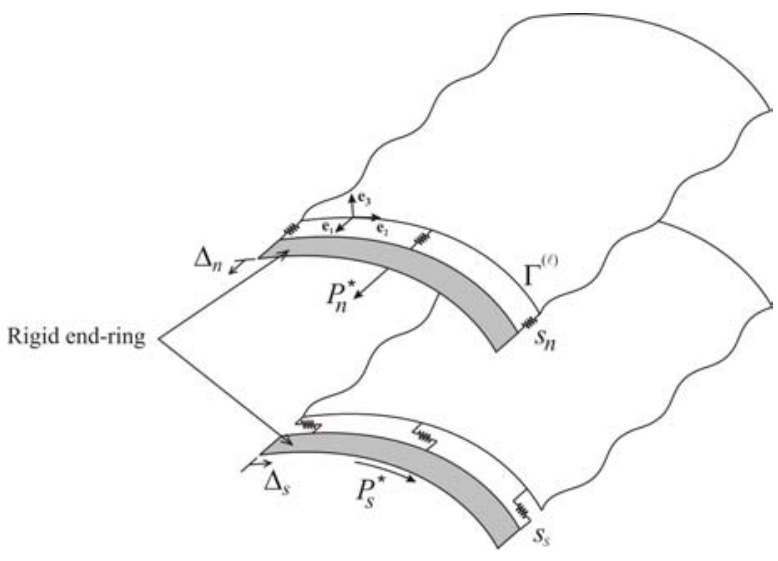

Fig. 3 Application of a concentrated force through a rigid end-ring by using elastic springs along a shell edge

\section{Prescribed edge displacements and rotations}

Edge displacements and rotations are applied to a shell by specifying values for the displacements and rotations shown in Fig. 4 that correspond to the positive-valued stress resultants shown in Fig. 1. In particular, the axial and tangential displacements, $u_{n}^{*}$ and $u_{s}^{*}$, respectively, that are applied to the $\ell^{\text {th }}$ boundary segment, $\Gamma_{(\ell)}$, are given by

$u_{1}\left(\mathbf{n} \cdot \mathbf{e}_{1}\right)=u_{n}^{*}$

$u_{2}\left[\left(\mathbf{e}_{3} \times \mathbf{n}\right) \cdot \mathbf{e}_{2}\right]=u_{s}^{*}$

Similarly, the transverse displacement $u_{3}^{*}$ and the rotation about an axis tangent to an edge $\vartheta_{n}^{*}$ that are applied to the $\ell^{\text {th }}$ boundary segment are defined by $u_{3}=u_{t}^{*}$

$u_{3,1}\left(\mathbf{n} \cdot \mathbf{e}_{1}\right)=\vartheta_{n}^{*}$
As mentioned previously, these prescribed displacements are enforced through the use of elastic edge restraints (springs) to relax kinematic admissibility requirments on the functions that are used to represent the displacement fields. The uniformly distributed extensional and rotational springs that are attached to the shell edges in the normal, tangential, and transverse directions and used to enforce the kinematic boundary conditions are depicted in Fig. 4.

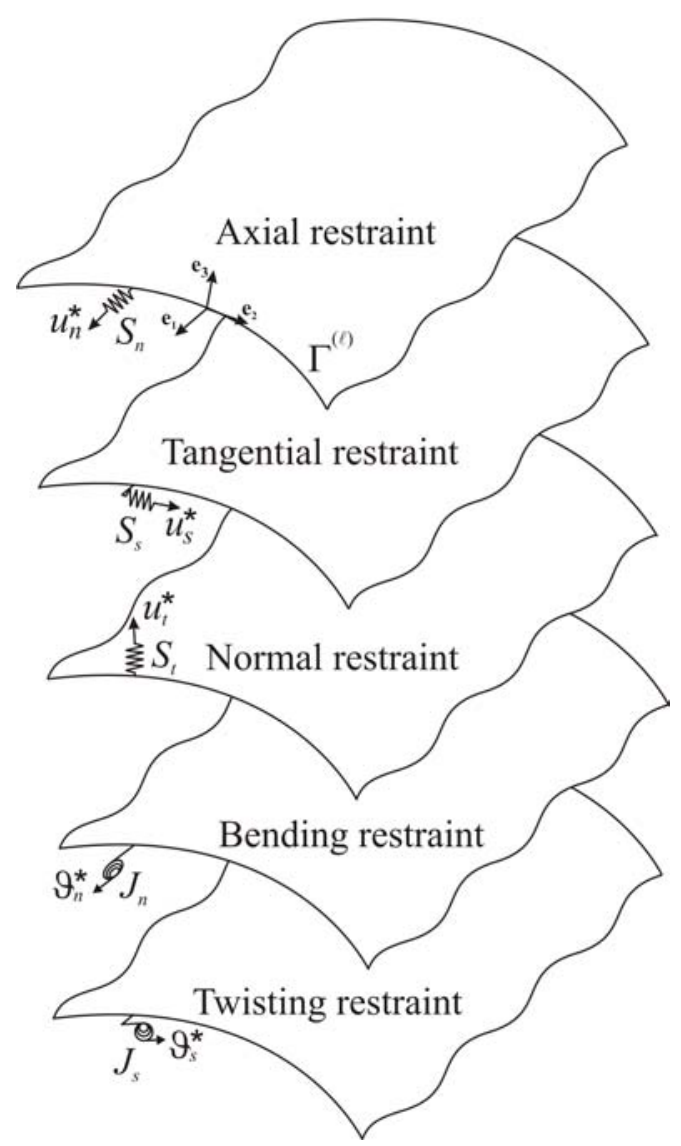

Fig. 4 Types of uniformly distributed elastic spring supports that can be prescribed along the edge of a cylindrical shell.

Specifying appropriate stiffness values for the springs results in full or partial restraints along the shell edges. A zero value of the spring stiffness corresponds to a traction-free-edge condition. In contrast, a value of the spring stiffness that is large compared to the corresponding shell stiffness effectively corresponds to a prescribed zero-valued boundary displacement or rotation. This approach effectively yields a prescribed kinematic boundary condition in the limit as the relative stiffness of the spring becomes much greater than the corresponding shell stiffness. Similarly, values for the spring stiffness can be selected that correspond to a specified uniform elastic restraint along an edge, similar 
to that provided by a rigid end-ring. This capability is important, and useful, because in some test fixtures or actual structures the edge supports may not be stiff enough to simulate a fully clamped boundary condition or flexible enough to simulate a simply supported boundary condition.

As depicted in Fig. 4, the membrane displacements, $u_{n}$ and $u_{s}$, and the transverse displacement, $u_{t}=u_{3}$ along the $\ell^{\text {th }}$ boundary segment are restrained by extensional springs with stiffness values of $S_{n}, S_{s}$, and $S_{t}$ in the directions normal, tangent, and transverse to the boundary, respectively. In addition to the extensional springs, the edge rotations, $\vartheta_{n}$ and $\vartheta_{s}$, along the $\ell^{\text {th }}$ boundary segment are restrained by rotational springs with stiffness values of $J_{n}$ and $J_{s}$ that correspond to rotation about axes tangent and normal to the edge, respectively.

Extensional springs in the directions normal and tangent to the shell edge, with stiffness values of $s_{n}$ and $s_{s}$, are also used to simulate load introduction through a rigid end-ring, as shown in Fig. 3. Specifying values for the spring stiffnesses $s_{n}$ and $s_{s}$ that are relatively large compared to the corresponding shell stiffnesses causes the shell edge to behave as if a rigid end-ring is attached that produces the uniformly distributed displacements with the corresponding magnitudes $\Delta_{n}$ and $\Delta_{s}$. The values for $\Delta_{n}$ and $\Delta_{s}$ that correspond to the specified concentrated loads are determined by using a penalty parameter approach. This approach enforces the difference between the edge displacements of the shell and the unknown uniform rigid end-ring displacements, $\left(u_{n}-\Delta_{n}\right)$ and $\left(u_{s}-\Delta_{s}\right)$ to vanish, while retaining the corresponding potential energy of the applied concentrated loads $P_{n}^{*}$ and $P_{s}^{*}$.

\section{Kinematics and Stress-Strain Relations}

The kinematic equations used in the present study are based, to a large extent, on the assumptions of Love-Kirchhoff classical thin-shell theory. Specifically, the axial, circumferential (tangential), and normal (normal to the mid-surface) displacements of a generic point of the shell are denoted by $U_{1}\left(s_{1}, s_{2}, s_{3}\right)$, $U_{2}\left(s_{1}, s_{2}, s_{3}\right)$ and $U_{3}\left(s_{1}, s_{2}, s_{3}\right)$, respectively. The corresponding displacements of a generic point of the shell mid-surface that share the same unit vector normal to the mid-surface are denoted by $u_{1}\left(s_{1}, s_{2}\right), u_{2}\left(s_{1}, s_{2}\right)$ and $u_{3}\left(s_{1}, s_{2}\right)$, respectively. In classical shell theory, these displacements are related by

$$
\begin{aligned}
& U_{1}\left(s_{1}, s_{2}, s_{3}\right)=u_{1}\left(s_{1}, s_{2}\right)-s_{3} \beta_{1}\left(s_{1}, s_{2}\right) \\
& U_{2}\left(s_{1}, s_{2}, s_{3}\right)=u_{2}\left(s_{1}, s_{2}\right)-s_{3} \beta_{2}\left(s_{1}, s_{2}\right)
\end{aligned}
$$

$U_{3}\left(s_{1}, s_{2}, s_{3}\right)=u_{3}\left(s_{1}, s_{2}\right)(10 \mathrm{c})$

where $\beta_{1}\left(s_{1}, s_{2}\right)$ and $\beta_{2}\left(s_{1}, s_{2}\right)$ are the mid-surface rotations about the $s_{2}$ and $s_{1}$ axes, respectively, that are given by

$\beta_{1}\left(s_{1}, s_{2}\right)=u_{3,1}\left(s_{1}, s_{2}\right)$

$\beta_{2}\left(s_{1}, s_{2}\right)=u_{3,2}\left(s_{1}, s_{2}\right)-\frac{1}{R\left(s_{2}\right)} u_{2}\left(s_{1}, s_{2}\right)$

in which a subscript after a comma denotes partial differentiation. The corresponding linear membranestrain-displacement relations are given by

$$
\boldsymbol{\varepsilon}=\left\{\begin{array}{l}
\varepsilon_{11} \\
\varepsilon_{22} \\
\gamma_{12}
\end{array}\right\}=\left\{\begin{array}{c}
u_{1,1} \\
\left(u_{2,2}+\frac{1}{R} u_{3}\right) \\
\left(u_{1,2}+u_{2,1}\right)
\end{array}\right\}
$$

and the bending-strain-displacement relations are given by

$$
\mathbf{\kappa}=\left\{\begin{array}{l}
\kappa_{11} \\
\kappa_{22} \\
\kappa_{12}
\end{array}\right\}=\left\{\begin{array}{c}
-u_{3,11} \\
-\left(u_{3,22}-\left(\frac{u_{2}}{R}\right)_{, 2}\right) \\
-2\left(u_{3,12}-\frac{1}{R} u_{2,1}\right)
\end{array}\right\}
$$

It is important to point out that the expression given for the change in surface twist due to deformation, $\kappa_{12}$, is that originally published by Love ${ }^{26,27}$ in 1888 for general shells, in terms of lines of principal-curvature coordinates, and derived in the book by Timoshenko and Woinowsky-Krieger ${ }^{28}$ for circular cylindrical shells. As indicated by Bushnell, ${ }^{29}$ the expression for $\kappa_{12}$ vanishes for rigid-body motions in contrast to the corresponding expression presented in Reissner's version of Love's first-approximation shell theory (see Reissner, ${ }^{30}$ Kraus, ${ }^{31}$ and Naghdi ${ }^{32}$ ). Equations (12a) and (12b), and the more general forms presented by Bushnell, ${ }^{29}$ are sometimes referred to as the LoveTimoshenko strain-displacement equations. Justification for this terminology is given by Chaudhuri. $^{33}$

The stress-strain relations used in the present study are those of the classical theory of laminated plates and shells, ${ }^{34}$ which are based on a linear through-thethickness distribution of the strain fields. For a thin, symmetrically laminated cylindrical shell, with variable wall thickness, the relationship between the membrane and bending stress resultants and the membrane and bending strains is expressed conveniently in matrix notation by 


$$
\mathbf{N}=\mathbf{A}\left(s_{1}, s_{2}\right) \boldsymbol{\varepsilon}
$$

and

$$
\mathbf{M}=\mathbf{D}\left(s_{1}, s_{2}\right) \boldsymbol{\kappa}
$$

The membrane and bending stress resultants in Eqs. (13a) and (13b) are defined as

$$
\mathbf{N}^{T}=\left\{N_{11}, N_{22}, N_{12}\right\}
$$

and

$$
\mathbf{M}^{T}=\left\{M_{11}, M_{22}, M_{12}\right\}
$$

It is important to reiterate that when shell-wall thickness variations are present, the membrane and bending stiffness matrices, $\mathbf{A}\left(s_{1}, s_{2}\right)$ and $\mathbf{D}\left(s_{1}, s_{2}\right)$, are dependent on the curvilinear surface coordinates $s_{1}$ and $s_{2}$.

It is convenient, in the present study, to combine the relations given in Eqs. (13a) and (13b) into the matrix form

$$
\mathbf{s}=\mathbf{C e}
$$

in which $\mathbf{s}$, $\mathbf{e}$ and $\mathbf{C}$ are defined as follows:

$$
\begin{aligned}
& \mathbf{s}^{T}=\left\{\mathbf{N}^{T}, \mathbf{M}^{T}\right\} \\
& \mathbf{e}^{T}=\left\{\boldsymbol{\varepsilon}^{T}, \boldsymbol{\kappa}^{T}\right\} \\
& \mathbf{C}=\mathbf{C}\left(s_{1}, s_{2}\right)=\left[\begin{array}{cc}
\mathbf{A}\left(s_{1}, s_{2}\right) & \mathbf{0} \\
\mathbf{0} & \mathbf{D}\left(s_{1}, s_{2}\right)
\end{array}\right]
\end{aligned}
$$

\section{Equations Governing the Response}

A general analytical approach for the exact solution of the equilibrium equations for a laminated-composite cylindrical shell with variable curvature is not mathematically tractable. Therefore, a semi-analytic variational approach that is based on the principle of stationary potential energy is used in the present study to obtain numerical results. Because elastic edge retraints are used as a means to relax the kinematic admissability conditions on the assumed displacement functions, and because a rigid-end-ring capability is used to impose shell-end force resultants, the potential energy consists of the elastic strain energy of the shell and the elastic edge restraints and the potential energy of the applied loads. In particular, the potential energy is expressed symbolically by

$$
\pi(\mathbf{q}, \Delta)=U(\mathbf{q})+\Omega(\mathbf{q}, \Delta)+V(\mathbf{q}, \Delta)
$$

in which $U$ and $\Omega$ represent the strain energy of the laminate and the elastic edge supports (springs), and $V$ represents the potential energy due to external boundary loads. Their explicit forms are presented in Appendix A. The symbol $\mathbf{q}$ is the vector of unknown, generalized displacement coefficients that arises from the mathematical representation of the mid-surface displacement fields that is used in the variational solution process. In particular, the mid-surface displacement fields are given symbolically by $u_{1}(\mathbf{q})$, $u_{2}(\mathbf{q})$, and $u_{3}(\mathbf{q})$. The symbol $\Delta$ represents the vector of unknown edge displacements that arise from prescribing end loads.

Subjected to the constraint equations that arise from the use of Lagrange multipliers, the equations governing the shell response are obtained by enforcing the requirement that the first variation of the total potential energy vanish. As discussed by McFarland et al., ${ }^{35}$ because the constraint equations are not functionally dependent on spatial coordinates, $s_{1}$ and $s_{2}$, the equations governing the response may be generated by modifying the total potential energy into the form

$\pi^{*}(\mathbf{q}, \Delta, \lambda)=\pi(\mathbf{q}, \Delta)+W(\mathbf{q}, \lambda)$

in which $W$ is viewed as the potential energy arising from constraint reactions. In particular,

$W(\mathbf{q}, \boldsymbol{\lambda})=\boldsymbol{\lambda}^{T} \mathbf{G} \mathbf{q}=0$

where $\lambda$ is the unknown vector of Lagrange multipliers and $\mathbf{G}$ is the known constraint coefficient matrix.

Substituting the specific expressions for $U(\mathbf{q})$, $\Omega(\mathbf{q}, \Delta), \quad V(\mathbf{q}, \Delta)$, and $W(\mathbf{q}, \lambda)$ that arise from approximation of the surface-displacement field and enforcing the first variation of the modified form of the total potential energy to vanish lead to

$$
\begin{aligned}
\delta \pi^{*} & =\delta \mathbf{q}^{T}\left[\mathbf{k}_{q q} \mathbf{q}+\mathbf{S}_{q q} \mathbf{q}-\mathbf{s}_{q \Delta} \Delta-\mathbf{f}^{*}-\mathbf{T}^{*}+\mathbf{G}^{T} \lambda\right] \\
& +\delta \Delta^{T}\left[\mathbf{s}_{\Delta \Delta} \Delta-\mathbf{s}_{q \Delta}^{T} \mathbf{q}-\mathbf{P}^{*}\right]+\delta \lambda^{T} \mathbf{G} \mathbf{q}=0
\end{aligned}
$$

in which the matrix, $\mathbf{k}_{q q}$ represents the stiffness matrix of the shell and requires evaluation of the corresponding integrand over a doubly connected region (see Appendix A for details). The springstiffness matrices, $\mathbf{S}_{q q}$ and $\mathbf{s}_{\Delta \Delta}$, are associated with the deformation of the shell edges and displacement of the rigid end-ring, respectively. The spring-stiffness matrix, $\mathbf{s}_{q \Delta}$, captures the coupling between the displacement of the shell edges and the rigid end-ring. The vectors $\mathbf{f}^{*}, \mathbf{T}^{*}$, and $\mathbf{P}^{*}$ arise from the prescribed boundary displacements, external tractions and moments, and the concentrated forces applied to a rigid end-ring, respectively. For the arbitrary variations $(\delta \mathbf{q}, \delta \Delta$, and $\delta \lambda)$, the stationary condition requires that the following equations must be satisfied:

$$
\begin{aligned}
& {\left[\left(\mathbf{k}_{q q}+\mathbf{S}_{q q}\right) \mathbf{q}-\mathbf{s}_{q \Delta} \Delta-\mathbf{f}^{*}-\mathbf{T}^{*}+\mathbf{G}^{T} \boldsymbol{\lambda}\right]=\mathbf{0}} \\
& {\left[\mathbf{s}_{\Delta \Delta} \Delta-\mathbf{s}_{q \Delta}^{T} \mathbf{q}-\mathbf{P}^{*}\right]=\mathbf{0}} \\
& \mathbf{G} \mathbf{q}=\mathbf{0}
\end{aligned}
$$


It is convenient, to express Eqs. (21a) - (21c) into the single matrix equation

\section{$\mathbf{K} \mathbf{Q}=\mathbf{F}$}

where $\mathbf{K}$ and $\mathbf{F}$ represent the overall, system stiffness matrix and the overall load vector, respectively. These matrices have the general, expanded form

$$
\mathbf{K}=\left[\begin{array}{ccc}
\mathbf{K}_{q q} & -\mathbf{s}_{q \Delta} & \mathbf{G}^{T} \\
-\mathbf{s}_{q \Delta}^{T} & \mathbf{s}_{\Delta \Delta} & \mathbf{0}^{T} \\
\mathbf{G} & \mathbf{0} & 0
\end{array}\right] \quad \text { and } \quad \mathbf{F}=\left\{\begin{array}{c}
\mathbf{F}^{*} \\
\mathbf{P}^{*} \\
0
\end{array}\right\}
$$

in which

$$
\mathbf{K}_{q q}=\mathbf{k}_{q q}+\mathbf{S}_{q q} \quad \text { and } \quad \mathbf{F}^{*}=\mathbf{f}^{*}+\mathbf{T}^{*}
$$

The vector of unknowns, $\mathbf{Q}$, that appears in Eq. (22) is defined as

$\mathbf{Q}=\left\{\begin{array}{l}\mathbf{q} \\ \Delta \\ \lambda\end{array}\right\}$

Solving for the vector of unknowns in Eq. (22) yields all the information needed to obtain a complete variational solution to a specific problem. The accuracy of a solution depends on the number of terms included in the expressions for the local and global functions representing the displacement fields and converges to the corresponding exact solution as the number of terms increases.

\section{Displacement-field representation}

Representation of the mid-surface displacement field is a critical step in the variational solution to the problem. By relaxing the requirements for kinematic admissibility, the mid-surface displacement fields are represented in the present study by a combination of rigid-body modes, $u_{R i}$, and global and local functions, denoted by $\bar{u}_{i}$ and $\overline{\bar{u}}_{i}$, respectively; that is,

$u_{i}=u_{R i}+\bar{u}_{i}+\overline{\bar{u}}_{i}$

where the values of the index are given by $i=1,2$, and 3 . The rigid-body modes account for the overall or global translation and rotation of the shell, and are selected so that they produce neither membrane strain nor changes in shell curvature and twist. These terms are included for the completeness of the kinematics of the cylindrical shell. The presence of the appropriate displacement boundary conditions inherently eliminates the rigid-body motion. However, for cases where an insufficient number of kinematic boundary conditions are imposed, these rigid-body terms need to be eliminated, as discussed in detail in Appendix $\mathrm{C}$. Following the complex-variable solution techniques used in the theory of elasticity, the local functions are expressed in terms of robust, uniformly convergent Laurent series (used for doubly connected regions) to enhance capturing steep stress gradients and deformations near the cutout. Complete sets of trigonometric expansions are used to primarily capture the overall global response of the shell. Here, completeness means that all the fundamental waveforms needed to construct the typical overall deformations of a shell are included in the set.

For convenience, the displacement representations are rewritten in matrix form as

$$
\begin{aligned}
& u_{i}=\mathbf{V}_{R i}^{T} \boldsymbol{\alpha}_{R}+\overline{\mathbf{V}}_{i}^{T} \mathbf{c}_{i}+\overline{\overline{\mathbf{V}}}_{i}^{T} \boldsymbol{\alpha} \quad(i=1,2) \\
& u_{3}=\mathbf{V}_{R 3}^{T} \boldsymbol{\alpha}_{R}+\overline{\mathbf{V}}_{3}^{T} \mathbf{c}_{3}+\overline{\overline{\mathbf{V}}}_{3}^{T} \boldsymbol{\beta}
\end{aligned}
$$

An even more useful, compact form is given by

$u_{i}=\mathbf{V}_{i}^{T} \mathbf{q}$ with $i=1,2,3$

where the vector of unknown displacement coefficients, $\mathbf{q}$, is defined by

$\mathbf{q}^{T}=\left\{\boldsymbol{\alpha}_{R}^{T}, \mathbf{c}_{1}^{T}, \mathbf{c}_{2}^{T}, \mathbf{c}_{3}^{T}, \boldsymbol{\alpha}^{T}, \boldsymbol{\beta}^{T}\right\}$

In Eq. (28), the vector $\boldsymbol{\alpha}_{R}$ contains the unknown coefficients for the rigid-body motion of the shell, and the vectors $\boldsymbol{\alpha}$ and $\boldsymbol{\beta}$ contain the real and imaginary parts of the unknown coefficients $\alpha_{n m}$ and $\beta_{n m}$, respectively, that are associated with the local functions. The vectors $\mathbf{c}_{i}$, where $i=1,2,3$, contain the real-valued unknown coefficients, $c_{i(m n)}$ that are associated with the global functions. The explicit forms used herein for the unknown coefficient vectors $\boldsymbol{\alpha}_{R}$, $\mathbf{c}_{i}, \boldsymbol{\alpha}$, and $\boldsymbol{\beta}$ that appear in Eqs. (26a) and (26b) along with the vector functions $\mathbf{V}_{i}$ (and the corresponding subvectors $\mathbf{V}_{R i}, \overline{\mathbf{V}}_{i}$, and $\overline{\overline{\mathbf{V}}}_{i}$ ) are given in Appendix B.

In addition to the general representation of the shell surface-displacement fields, similar matrix expressions are needed for the displacements and rotations of points on the shell boundary. In the present study, the boundary displacement vector $\mathbf{u}_{\Gamma}$ is introduced that consists of the mid-surface boundary displacements in the directions normal, tangent, and transverse to a shell edge, and the mid-surface rotations about axes that are normal and tangent to a shell edge. The boundary displacements in the directions normal, tangent, and transverse to a shell edge are denoted herein by $u_{n}, u_{s}$, and $u_{t}$, respectively. Similarly, the mid-surface rotations about axes that are tangent and normal to a shell edge are denoted by $\vartheta_{n}$ and $\vartheta_{s}$, respectively. In terms of the vector of unknowns defined by Eq. (28), the boundary displacements and rotations are expressed in matrix form by

$\mathbf{u}_{\Gamma}=\mathbf{B} \mathbf{q}$ 
in which the boundary displacement vector, $\mathbf{u}_{\Gamma}$ is defined by

$$
\mathbf{u}_{\Gamma}^{T}=\left\{u_{n}, u_{s}, u_{t}, \vartheta_{n}\right\}
$$

The matrix $\mathbf{B}$ is a known matrix of coefficients that is defined as

$$
\mathbf{B}=\left[\begin{array}{c}
\mathbf{u}_{n}^{T} \\
\mathbf{u}_{s}^{T} \\
\mathbf{u}_{t}^{T} \\
\boldsymbol{\theta}_{n}^{T}
\end{array}\right]
$$

in which the sub-vectors, $\mathbf{u}_{n}^{T}, \mathbf{u}_{s}^{T}, \mathbf{u}_{t}^{T}$ and $\boldsymbol{\theta}_{n}^{T}$ are known and defined by

$\mathbf{u}_{n}^{T}=\left(\mathbf{n} \cdot \mathbf{e}_{1}\right) \mathbf{V}_{1}$

$\mathbf{u}_{s}^{T}=\left[\left(\mathbf{e}_{3} \times \mathbf{n}\right) \cdot \mathbf{e}_{2}\right] \mathbf{V}_{2}$

$\mathbf{u}_{t}^{T}=\mathbf{V}_{3}^{T}$

and

$\boldsymbol{\theta}_{n}^{T}=\left(\mathbf{n} \cdot \mathbf{e}_{1}\right) \mathbf{V}_{3,1}^{T}$

\section{Strain- and stress-resultant-field representation}

After defining the shell mid-surface displacement field in terms of the generalized coordinate $\mathbf{q}$, the corresponding representation of the strains is obtained by substituting Eq. (27) into the strain-displacement relations given in vector form by Eqs. (12a) and (12b). This substitution yields

$\boldsymbol{\varepsilon}=\mathbf{L}_{\varepsilon} \mathbf{q}$

and

$$
\mathbf{\kappa}=\mathbf{L}_{\kappa} \mathbf{q}
$$

where the strain-coefficient matrices $\mathbf{L}_{\varepsilon}$ and $\mathbf{L}_{\kappa}$ are defined as

$$
\begin{aligned}
\mathbf{L}_{\varepsilon} & =\left[\begin{array}{c}
\mathbf{V}_{1,1}^{T} \\
\mathbf{V}_{2,2}^{T}+\frac{1}{R} \mathbf{V}_{3}^{T} \\
\mathbf{V}_{1,2}^{T}+\mathbf{V}_{2,1}^{T}
\end{array}\right] \\
\mathbf{L}_{\kappa} & =\left[\begin{array}{c}
-\mathbf{V}_{3,11}^{T} \\
-\mathbf{V}_{3,22}^{T}+\frac{1}{R} \mathbf{V}_{2,2}^{T}+\frac{R, 2}{R^{2}} \mathbf{V}_{2}^{T} \\
-2 \mathbf{V}_{3,12}^{T}+\frac{2}{R} \mathbf{V}_{2,1}^{T}
\end{array}\right]
\end{aligned}
$$

Next, the representations for $\boldsymbol{\varepsilon}$ and $\boldsymbol{\kappa}$ are substituted into Eq. (15b) to obtain

$$
\mathbf{e}=\mathbf{L} \mathbf{q}
$$

where the overall strain-coefficient matrix $\mathbf{L}$ is defined as

$$
\mathbf{L}^{T}=\left[\begin{array}{ll}
\mathbf{L}_{\varepsilon}^{T} & \mathbf{L}_{\kappa}^{T}
\end{array}\right]
$$

Finally, the corresponding matrix representation of the stress resultants in terms of the generalized coordinates is obtained by substituting Eq. (35) into constitutive Eq. (15). The resulting vector of stress resultants is given by

$\mathbf{s}=\mathbf{C} \mathbf{L} \mathbf{q}$

\section{Constraint Equations}

In the generalized-coordinate representations for $u_{1}$ and $u_{2}$, the coefficients $c_{1(00)}$ and $c_{2(00)}$ associated with the global functions, $\bar{u}_{1}$ and $\bar{u}_{2}$, also correspond to rigid-body translation in the $s_{1}$ direction and rigid-body rotation about the $s_{1}$ axis, respectively. These two redundant rigid-body modes are eliminated by introducing constraint conditions using Lagrange multipliers. In particular, the unknown Lagrange multipliers $\lambda_{R R B(1)}$ and $\lambda_{R R B(2)}$ are associated with the redundant rigid-body modes. Also, multi-valuedness of the normal-direction displacement $u_{3}\left(s_{1}, s_{2}\right)$ that arises from the presence of logarithmic terms in the Laurentseries-expansion for the local function-must be eliminated. The unknown Lagrange multipliers $\lambda_{S V(r)}$ and $\lambda_{S V(s)}$ are used herein to eliminate this multivaluedness. Likewise, the rigid-body modes of the cylindrical shell must be eliminated by the Lagrange multipliers $\lambda_{R B(j)}(j=1, . ., 6)$ if the specified kinematic boundary conditions are not sufficient enough to prevent them. In other words, the non-vanishing rigid body modes must be eliminated by introducing constraint conditions prior to the stress analysis in order for the overall system stiffness matrix $\mathbf{K}$, given in $\mathrm{Eq}$ (22), to be nonsingular.

These requirements on the representation of the shell displacement field are enforced by using constraint equations that use Lagrange multipliers. These constraint equations are functionally independent, forming a set of linearly independent equations equal in number to the total number of Lagrange multipliers. The Lagrange multipliers can be viewed as the reactions that are needed to enforce the corresponding constraints. In the present study, all of these constraint conditions are included in the matrix equation given in Eq. (19). The explicit form of the vector of unknown Lagrange multipliers, $\boldsymbol{\lambda}$, and the known coefficient matrix, $\mathbf{G}$, are given in Appendix C.

\section{Overview of Validation Studies}

A limited series of validation studies were conducted in the present study to determine the accuracy of results obtained by using analysis method presented herein. Specifically, the studies included circular and non- 
circular cylindrical shells with either a circular or an elliptical cutout under uniform tension. The stress resultants around the circular and elliptical cutout for varying aspect ratios and orientations in a circular cylinder as well as the stress concentrations arising from a circular cutout in a non-circular cylindrical shell were computed. Comparisons of the stress-resultant distributions and magnitudes in the shells were made with the corresponding results obtained by using an inhouse finite element program developed earlier by Madenci and Barut. ${ }^{36}$ This finite element program has been validated, to a large extent, against previously published experimental and numerical results for stress, buckling, and post-buckling of thin-shell structures (see Madenci and Barut ${ }^{37,38}$ ). Therefore, this finite element program is expected to serve as a reliable indicator of the accuracy of the analysis methods and results presented herein. Overall, the comparisons indicate very good agreement (less than $1 \%$ difference) between the corresponding results produced by the two analysis methods. For shells with high-aspect-ratio cutouts, differences of approximately 5\% were obtained and found to be the result of insufficient mesh refinement in the finite element models.

\section{Selected Numerical Results}

Selected numerical results are presented in this section to demonstrate the utility of the analysis method presented herein and the potential for its use in developing design technology. These results elucidate the effects of loading condition, non-circular crosssection geometry, wall-thickness variation, cutout shape, cutout size, and cutout orientation on the intensity of stress-resultant concentrations near a cutout. Specifically, tension, torsion, and pure-bending loads are considered for $\left[45^{0} /-45^{0} / 90^{\circ} / 0_{2}^{0} / 90^{\circ} /-45^{0} / 45^{0}\right]_{s}$ quasi-isotropic shells with length $L=356 \mathrm{~mm}$ and made of graphiteepoxy plies. The nominal ply thickness is $t_{k 0}=0.14 \mathrm{~mm}$, resulting in the total thickness of the shell given by $h=2.24 \mathrm{~mm}$, and the ply orientation angles are measured with respect to the longitudinal shell axis. The Young's moduli of each ply in the longitudinal, fiber direction and in the direction transverse to the fibers are specified as $E_{L}=135.0 \mathrm{GPa}$ and $E_{T}=13.0 \mathrm{GPa}$, respectively. The in-plane shear modulus and Poisson's ratio of each ply are given by $G_{L T}=6.4 \mathrm{GPa}$ and $v_{L T}=0.38$.

The effects of varying the radius of curvature $R_{0}$ on the stress-resultant concentration along the contour of a circular cutout with radius $a=25.5 \mathrm{~mm}$ are shown in Fig. 5 for a circular cylindrical shell subjected to a uniform axial tension load. Four curves that correspond to values of $R_{0} / L=0.5,0.75 .1$, and 1.25 are presented that show the tangential stress resultant, $N_{\phi \phi}$ normalized by the far-field applied uniform stress resultant $N_{0}$, as a function of position around the cutout (indicated by the "cutout angle", $\phi$ ). As shown in Fig. 5 , the stress-resultant concentration is a maximum at $\phi=90^{\circ}$ and $270^{\circ}$ (at the net section of the shell) for each case and reduces from a maximum value of approximately 4.0 to a minimum value of 3.4 at the net section as the radius of curvature increases. In addition, the results show that the $N_{\phi \phi}\left(a, 90^{\circ}\right)$ stress-resultant concentration approaches the well-known value of three for an isotropic plate as the shell radius increases. Away from the net section, changes in the radius of curvature have a relatively small effect on the stress-resultant concentration.

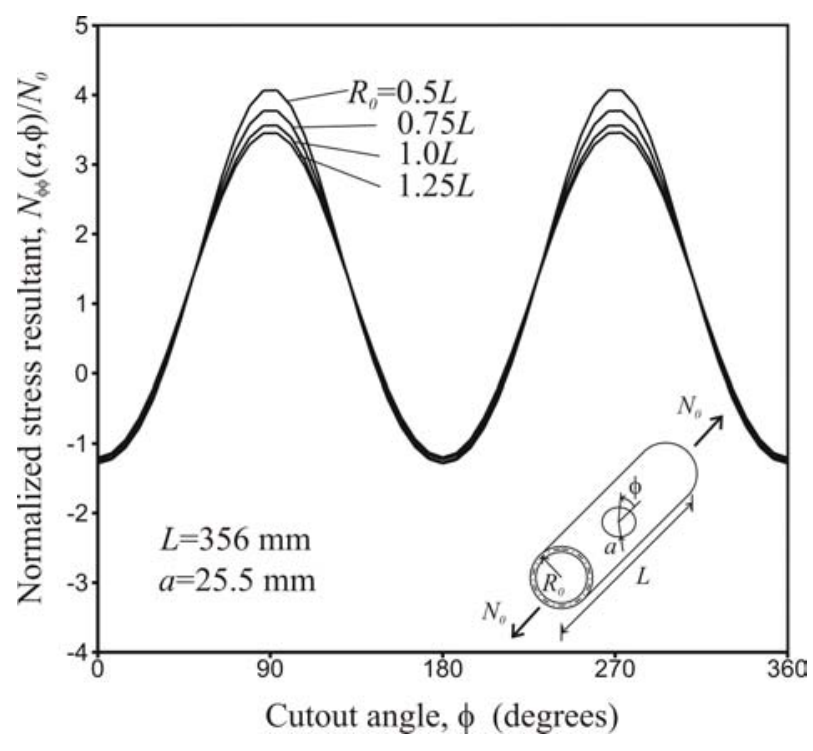

Fig 5 The effect of varying shell radius on the tangential stress resultants around a circular cutout in a quasiisotropic circular cylindrical shell subjected to a uniform tension load.

The effects of varying the circular-cutout radius on the stress-resultant concentration along the contour of a circular cutout is shown in Fig. 6 for a circular cylindrical shell with radius $R_{0}=381 \mathrm{~mm}$ and subjected to a uniform axial tension load. Five curves that correspond to values of the cutout radius $a=15$, 25.5. 30,40 , and $50 \mathrm{~mm}$ are presented that also show the tangential stress resultant $N_{\phi \phi}(a, \phi)$, normalized by the far-field applied uniform stress resultant, $N_{0}$, as a function of the cutout angle $\phi$. The results in Fig. 6 show that the stress-resultant concentration is a maximum at the net section of the shell for each case, 
as expected, and changes significantly from a minimum value of approxiamtely 3.1 to a maximum value of 5.1 at the net section as the cutout radius increases - an increase of approximately $65 \%$. The results also show that the $N_{\phi \phi}\left(a, 90^{\circ}\right)$ stress-resultant concentration approaches the well-known value of three for an isotropic plate as the cutout radius decreases. Away from the net section, changes in the cutout radius have a much smaller effect on the stress-resultant concentration.

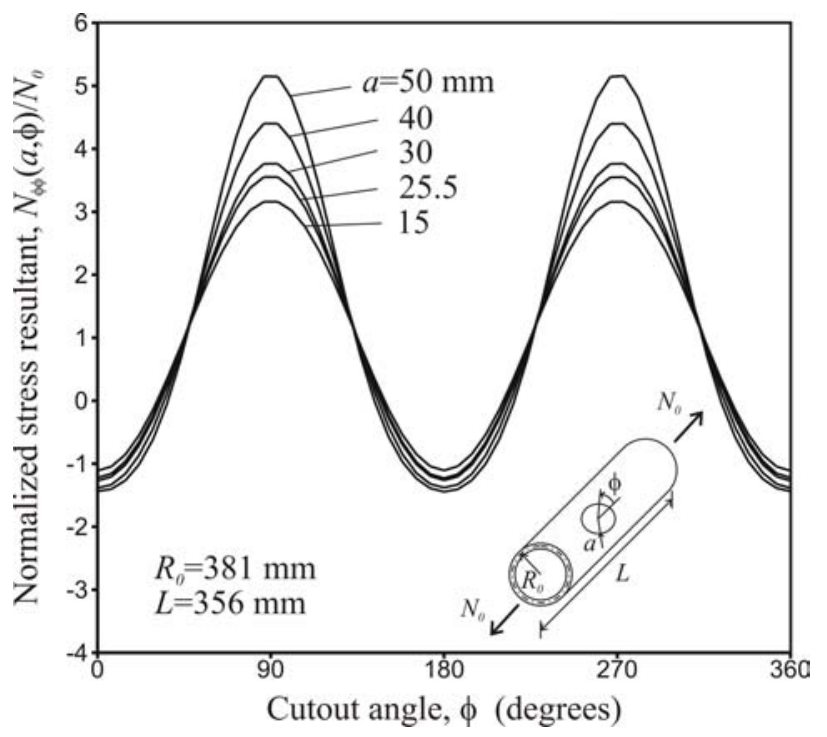

Fig 6 The effect of varying circular-cutout radius on the tangential stress resultants around a circular cutout in a quasi-isotropic circular cylindrical shell subjected to a uniform tension load.

The effect of varying the elliptical-cutout aspect ratio, $a / b$, on the tangential stress-resultant distribution around the edge of a cutout in a cylindrical shell with radius $R_{0}=178 \mathrm{~mm}$, and subjected to uniform tension is presented in Fig. 7. The orientation of the elliptical cutout is specified by $\psi=0^{0}$. Two curves that correspond to the locations $\phi=0^{\circ}$ and $90^{\circ}$ are presented that show the tangential stress resultant, $N_{\beta \beta}\left(\alpha_{0}, \beta\right)$ normalized by the far-field applied uniform stress resultant $N_{0}$, as a function of the cutout aspect ratio. As expected, the normalized stress-resultant concentration, $N_{\beta \beta}\left(\alpha_{0}, \beta\right) / N_{0}$, remains negative for all aspect ratios at $\phi=0^{\circ}$, consistent with the expected Poisson effect, and the magnitudes are relatively insignificant at this location. In contrast, large stressresultant concentrations are indicated at the net section $\left(\phi=90^{\circ}\right)$ that diminish from a maximum value of approximately 17.0 for a widthwise, slot-like cutout with $(a=5 \mathrm{~mm}$ and $b=30 \mathrm{~mm})$ or $(a / b=1 / 6)$ to a minimum value of 1.4 for a lengthwise, slot-like cutout $(a=30 \mathrm{~mm}$ and $b=5 \mathrm{~mm})$ or $(a / b=6)$.

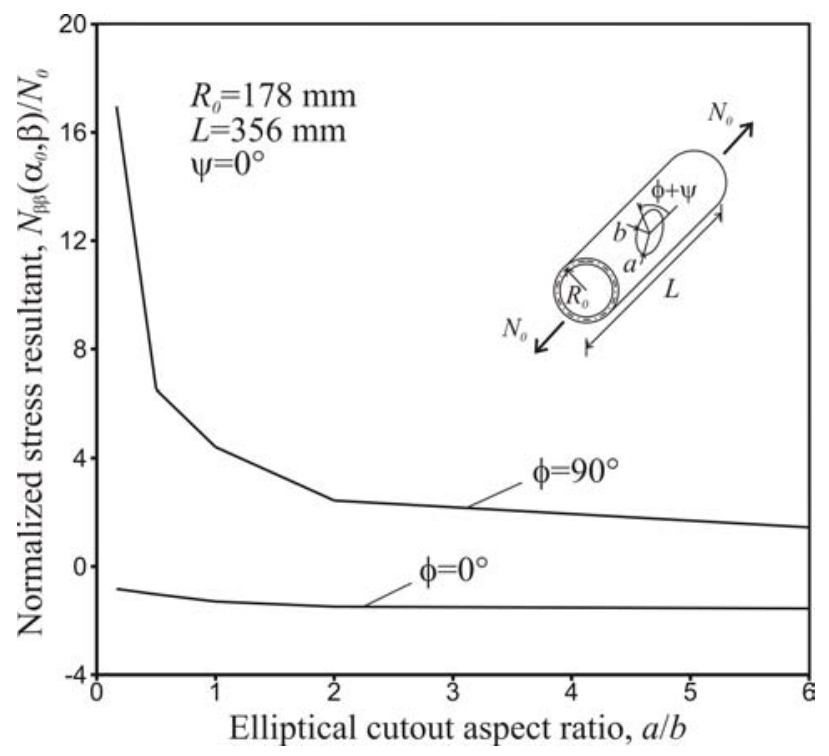

Fig 7 The effect of varying elliptical-cutout aspect ratio on the tangential stress resultants around a cutout in a quasi-isotropic cylindrical shell subjected to a uniform tension load.

The effects of varying the orientation of a highaspect-ratio, slot-like elliptical cutout on the stressresultant concentration along the cutout contour is shown in Fig. 8 for a circular cylindrical shell with radius $R_{0}=178 \mathrm{~mm}$ and subjected to a uniform axial tension load. The major and minor axes of the cutout are given by $a=30 \mathrm{~mm}$ and $b=5 \mathrm{~mm}$, respectively. The orientation of the elliptical cutout, with respect to the longitudinal shell axis, is measured by the angle, $\psi$. Three curves that correspond to values of $\psi=0^{0}, 45^{\circ}$, and $90^{\circ}$ are presented that show the tangential stress resultant at the cutout edge, $N_{\beta \beta}$ normalized by the farfield applied uniform stress resultant, $N_{0}$ as a function of the cutout angle $\phi$.

The results in Fig. 8 show that the stress-resultant concentration is the least pronounced for the case of $\psi=0^{0}$. For this case, the cutout major axis is aligned lengthwise with the shell axis and the net section of the shell is the largest. The location on the cutout edge defined by $\phi=0^{0}$ corresponds to where the edge of the cutout intersects the major axis. At this location, the edge of the cutout is in tangential compression $\left(N_{\beta \beta} / N_{0}=-1.6\right)$, consistent with a Poisson effect.

The location defined by $\phi=90^{\circ}$ corresponds to where 
the edge of the cutout intersects the minor axis; that is, at the net section of the shell. At this location, the edge of the cutout is in tangential tension $\left(N_{\beta \beta} / N_{0}=1.4\right)$. Between approximately $\phi=10^{\circ}$ and $170^{\circ}$ and between $\phi=190^{\circ}$ and $350^{\circ}$, the cutout width (and hence net section width) does not vary greatly. This attribute accounts for the corresponding flat regions in the $\psi=0^{0}$ curve shown in Fig. 8 .

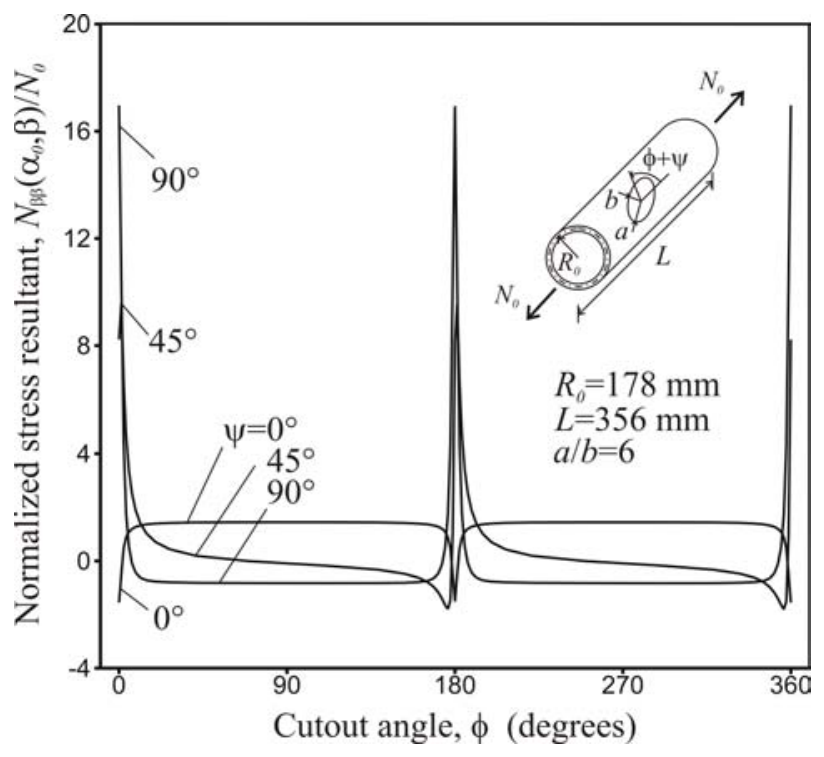

Fig 8 The effect of varying elliptical-cutout orientation on the tangential stresses around the cutout in a quasiisotropic cylindrical shell subjected to a uniform tension load.

For the case of $\psi=90^{\circ}$, the cutout major axis is perpendicular to the shell axis and the net section of the shell is the smallest. As before, the locations defined by $\phi=0^{\circ}$ and $180^{\circ}$ correspond to where the edge of the cutout intersects the major axis; that is, at the net section of the shell. The results in Fig. 9 show that the edge of this high-aspect-ratio cutout has extremely high stress-resultant concentrations at these locations $\left(N_{\beta \beta} / N_{0}=17\right.$.) that have very step gradients. Between approximately $\phi=5^{0}$ and $175^{\circ}$ and between $\phi=185^{\circ}$ and $355^{\circ}$, the analysis predicts relatively benign variations in the stress-resultant concentration. The case of $\psi=45^{\circ}$, exhibits stress-resultant concentrations that are, for the most part, bounded by the corresponding results for $\psi=0^{\circ}$ and $90^{\circ}$. The analysis also predicts very high stress-resultant concentrations where the cutout edge intersects the major principal cutout axis $\left(N_{\beta \beta} / N_{0}=8.2\right)$.
The effects of varying the cross-section eccentricity (see Eq. (2)) of a tension-loaded oval shell with a circular cutout are shown in Fig. 9. The results in this figure correspond to the equivalent shell radius $R_{0}=381 \mathrm{~mm}$ and a circular-cutout radius given by $a=25.5 \mathrm{~mm}$. Moreover, the tangential stress-resultant concentation at the shell net section, $N_{\phi \phi}\left(a, 90^{\circ}\right)$, normalized by the applied $\operatorname{load} N_{0}$, is shown as a function of the eccentricity parameter for the range of $-0.15 \leq \xi \leq 0.15$. As indicated in the figure, negative and positive values of $\xi$ correspond to cylindrical shells with the largest cross-sectional width oriented parallel and perpendicular to the tangent plane that passes through the two points of the cutout edge that are on the surface generator that passes through the center of the cutout, respectively. A value of $\xi=0$ corresponds to a circular cross-section and a value of $\xi=0.15$ corresponds to cross-sectional aspect ratio of 0.9 .

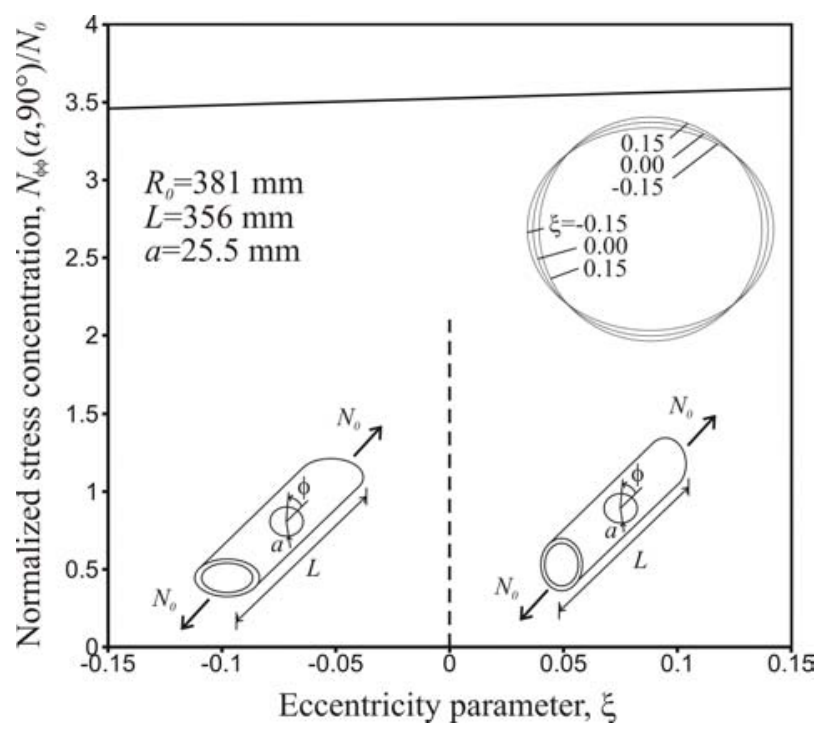

Fig 9 The effect of shell cross-section eccentricity on the stress-resultant concentration in an oval quasiisotropic cylindrical shell with a circular cutout and subjected to a uniform tension load.

The results presented in Fig. 9 show that the stressresultant concentration is affected benignly by the cross-sectional eccentricity. In particular, the stressresultant concentration increases almost linearly with increases in the eccentricity parameter from $N_{\phi \phi}\left(a, 90^{\circ}\right) / N_{0}=3.5$ to 3.6 , which is slightly less than a $3 \%$ variation. This trend is understood by noting that the shells that correspond to negative values of $\xi$ are flatter near the cutout than those that correspond to 
positive values of $\xi$ and, as indicated by the results in Fig. 5, are expected to have the lower values for the stress-resultant concentrations.

The effects of longitudinal and circumferential periodic wall-thickness variations on the stress-resultant concentration at the net section of circular cylindrical shell with radius $R_{0}=178 \mathrm{~mm}$, circular cutout radius $a=25.5 \mathrm{~mm}$, and subjected to uniform axial tension load are shown in Fig. 10. Two monotonically increasing curves that correspond to values of $\varepsilon_{1}$ (with $\varepsilon_{2}=0$ ) and $\varepsilon_{2}$ (with $\varepsilon_{1}=0$ ) are presented that show the tangential stress resultant $N_{\phi \phi}\left(a, 90^{\circ}\right)$, normalized by the far-field applied uniform stress resultant $N_{0}$, as a function of thickness-variation amplitudes (see Eq.(3)) that range from 0 to 0.2. For the longitudinal thickness variation, the wave numbers used in Eq. (3) are $m_{1}=1$ and $m_{2}=0$. Similarly, for the circumferential thickness variation, the wave numbers used in Eq. (3) are $m_{1}=0$ and $m_{2}=1$.

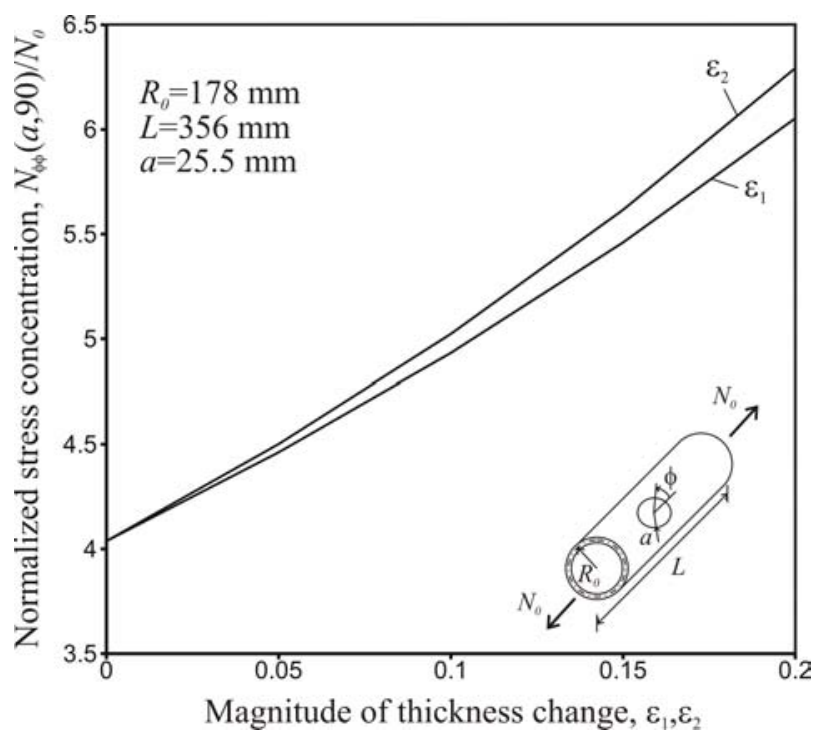

Fig 10 The effects of longitudinal $\left(\varepsilon_{1} \neq 0\right.$ and $\left.\varepsilon_{2}=0\right)$ and circumferential ( $\varepsilon_{1} \neq 0$ and $\varepsilon_{2}=0$ ) wall thickness variations on the tangential stress-resultant concentration around a circular cutout in a quasiisotropic circular cylindrical shell subjected to a uniform tension load.

The results shown in Fig. 10 indicate that the stressresultant concentration at the shell net section increases as the magnitude of the thickness variation increases, for variations in either the longitudinal or circumferential direction. The maximum variation in the results is approximately $56 \%$. Furthermore, the change in the stress-resultant concentration is slightly more pronounced for the circumferential thickness variation than for the longitudinal thickness variation. These increases are primarily due to a drastic loss of bending stiffness near the net section of the shell, as indicated by the wave numbers $m_{1}=0$ and $m_{2}=1$, where the thickness of the shell near the center of the cutout is smaller.

The effects of varying the radius of curvature $R_{0}$ on the stress-resultant concentration along the contour of a circular cutout with radius $a=25.5 \mathrm{~mm}$ is shown in Figs. 11 and 12 for a circular cylindrical shell subjected to a uniform torsion load and a pure-bending load, respectively. The pure-bending load corresponds to using $t_{n}^{*}=M_{0} \cos \left(\pi / s_{2}\right)$ in Eq. (5a). Four curves that correspond to values of $R_{0} / L=0.5,0.75$. 1 , and 1.25 are presented that show the normalized values of the tangential stress resultant $N_{\phi \phi}$ as a function of position around the cutout. In Fig. 11, $N_{\phi \phi .}$ is normalized by the far-field applied uniform shear stress resultant, $T_{0}$. In Fig. 12, $N_{\phi \phi}$ is normalized by the far-field applied uniform bending stress resultant, $\mathrm{M}_{\mathrm{o}}$.

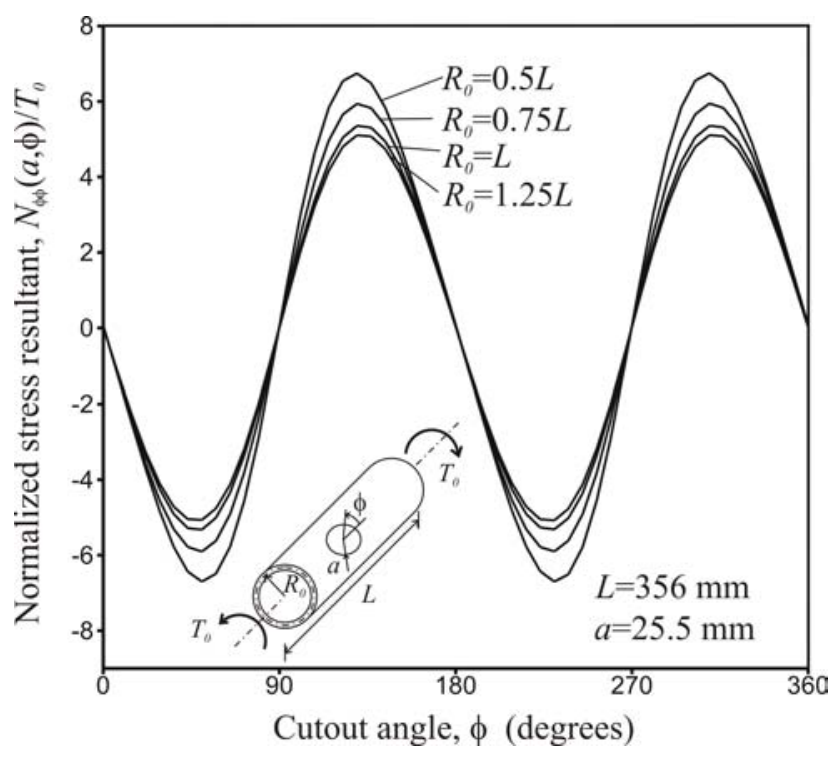

Fig 11 The effect of varying shell radius on the tangential stresses around a circular cutout in a quasi-isotropic circular cylindrical shell subjected to a uniform torsion load.

The results in Fig. 11 indicate that the stressresultant concentration has identical maximum magnitudes at $\phi=45^{0}, 135^{\circ}, 225^{\circ}$, and $315^{\circ}$ (at the net section of the shell) for each case, which corresponds to maximum diagonal tension and compression stress resultants associated with the shear stress resultants near the cutout. The magnitudes of the stress-resultant 
concentration for these four locations reduces from a maximum value of 6.8 to a minimum value of 5.1 as the radius of curvature increases (33\% variation). Away from these four locations, changes in the radius of curvature have a smaller effect on the stress-resultant concentration. The results in Fig. 12 indicate that the stress-resultant concentration for the shell subjected to the pure-bending load is quite similar to that presented in Fig. 5 for the corresponding tension-loaded shell.

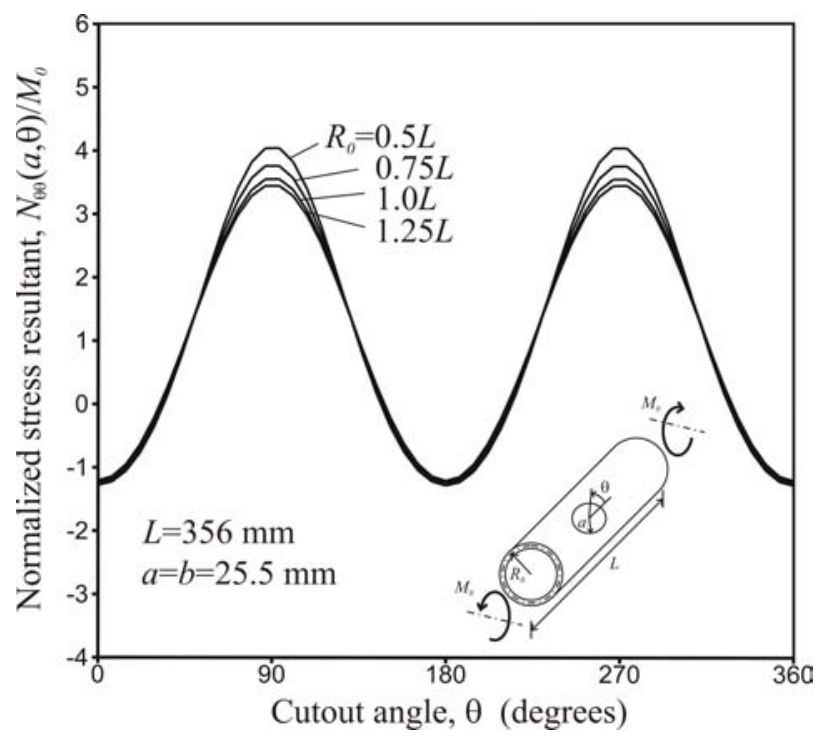

Fig 12 The effect of varying shell radius on the tangential stresses around a circular cutout in a quasi-isotropic circular cylindrical shell subjected to a purebending load.

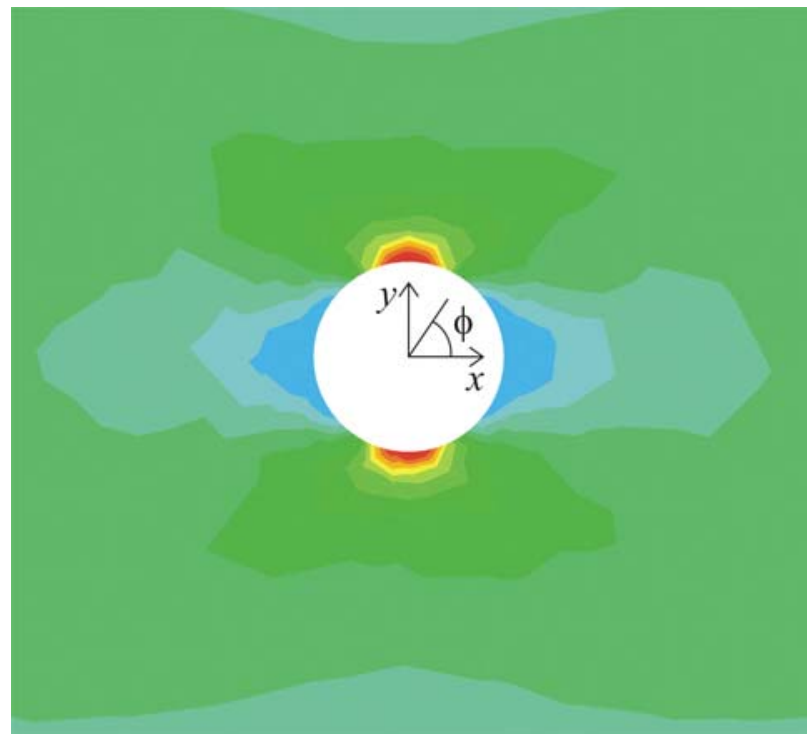

Fig 13 Stress resultant distribution near the cutout in a quasiisotropic circular cylindrical shell subjected to a pure-bending load.
Specifically, the stress-resultant concentration is a maximum at $\phi=90^{\circ}$ and $270^{\circ}$ (at the net section of the shell) for each case and reduces from a maximum value of 4.0 to a minimum value of 3.5 at the net section as the radius of curvature increases (14\% variation). In addition, $N_{\phi \phi} / M_{0}$ approaches the well-known value of three for an isotropic plate as the shell radius increases, and away from the net section, changes in the radius of curvature have a relatively small effect on the stressresultant concentration. For the case of $R_{0} / L=0.5$ shown in Fig. 12, a contour plot of $N_{\phi \phi} / M_{0}$ near the cutout is shown in Fig. 13. The extent of the stress concentration at the shell net section $\left(\phi=90^{\circ}\right.$ and $\left.270^{\circ}\right)$ is clearly captured by the analysis method presented herein. The highest stress-resultant concentration is $N_{\phi \phi}\left(a, 90^{\circ}\right) / M_{0}=4$ and it attenuates to the value of 1.01 at a radius of about $80 \mathrm{~mm}$ (approximately three times the cutout radius), measured from the center of the cutout.

\section{Concluding Remarks}

A special-purpose, semi-analytical approach based on complex potential functions has been presented that can be used to investigate the behavior of thin, noncircular cross-section cylindrical shells made of laminated-composite materials and with a cutout, efficiently and parametrically. In particular, the effects of radius of curvature; elliptical cutout size, aspect ratio, and orientation; oval cross-section eccentrictity; wall-thickness variations; and loading conditions on the stress-resultant concentration near the cutout have been presented for a quasi-isotropic shell subjected to uniform tension, uniform torsion and pure bending. In addition, studies that were conducted to validate the analysis method have been described.

A key finding of the results obtained with this analysis method is that the maximum tangential stressresultant concentration near a circular cutout in a tension-loaded, circular, quasi-isotropic shell increases by approximately $18 \%$ as the shell radius-to-length ratio decreases from 1.25 to 0.5 . Likewise, increases in the maximum tangential stress-resultant concentration as large as $65 \%$ have been found to occur with a fivefold increase in cutout radius. Results have also been presented that show extremely high tangential stressresultant concentrations can occur for high aspect ratio elliptical cutouts whose principal axes are not aligned with the longitudinal axis of a tension-loaded shell.

Additionally, results have been presented that show tension-loaded oval shells with a circular cutout on one of the flatter sides exhibit slightly lower tangential stress-resultant concentrations than the corresponding shell with the cutout on one of the more highly curved sides. Results have also been presented that show that 
wall-thickness variations in either the longitudinal or circumferential directions significantly affect the stress concentration, with respect to that for the corresponding shell with a nominal thickness. The analysis also predicts that a quasi-isotropic shell with a circular cutout and subjected to pure bending that yields the maximum tensile stress resultant at the longitudinal axis of the cutout behaves similarly to the corresponding tension-loaded shell. The corresponding shell subjected to torsion was found to exhibit the maximum tangential stress-resultant concentrations at locations consistent with the maximum diagonal tension and compression near the cutout. Overall, the results demonstrate that the analysis approach is a powerful means for developing design criteria for laminated-composite shells.

\section{Acknowledgement}

The authors wish to dedicate this paper to the memory of Dr. James H. Starnes, Jr. of the NASA Langley Research Center. Dr. Starnes was an internationally recognized expert in aerospace structures technology and a proponent of the development of special-purpose, design-oriented analysis methods such as that presented herein.

\section{References}

${ }^{1}$ Lurie, A. I., "Concentration of Stresses in the Vicinity of an Aperture in the Surface of a Circular Cylinder," Prikl. Mat. Mekh., Vol. 10, 1946, pp. 397406.

${ }^{2}$ Lurie, A. I., Statics of Thin-Walled Elastic Shells, State Publishing House of Technical and Theoretical Literature, Moscow, 1947.

${ }^{3}$ Lekkerkerker, J. G., "Stress Concentration Around Circular Holes in Cylindrical Shells," AIAA Journal, Vol. 10, 1964, pp. 1466-1472.

${ }^{4}$ Van Dyke, P., "Stresses about a Circular Hole in a Cylindrical Shell," AIAA Journal, Vol. 3, 1965, pp. 1733-1742.

${ }^{5}$ Ashmarin, I. A., "Stress Concentration Around a Circular Opening in an Orthotropic Cylindrical Shell," Prikladnaya Mekhanika, Vol. 2, 1966, pp. 44-48.

${ }^{6}$ Murthy, M. V. V., Rao, K. P. and Rao, A. K., "On the Stress Problem of Large Elliptical Cutouts and Cracks in Circular Cylindrical Shells," International Journal of Solids and Structures, Vol. 10, 1974, pp. 1243-1269.

${ }^{7}$ Guz, A. N., Chernyshenko, I. S. and Shnerenko, K. I., "Stress Concentration Near Openings in Composite Shells," International Applied Mechanics, Vol. 37, 2001, pp. 139-181.

${ }^{8}$ Van Tooren, M. J. L., Van Stijn, I. P. M. and Beukers, A., "Curvature Effects on the Stress Distribution in Sandwich Cylinders with a Circular Cutout," Composites: Part A, Vol. 3, 2002, pp. 1557-1572.
${ }^{9}$ Tennyson, R.C., "The Effects of Unreinforced Circular Cutouts on the Buckling of Circular Cylindrical Shells under Axial Compression," ASME Journal of Engineering Industry, Vol. 90, 1968, pp. 541-546.

${ }^{10}$ Starnes, J. H., Jr., "Effect of a Circular Hole on the Buckling of Cylindrical Shells Loaded by Axial Compression," AIAA Journal, Vol. 10, 1972, pp. 14661472 .

${ }^{11}$ Pierce, D. N. and Chou, S. I., "Stresses Around Elliptical Holes in Circular Cylindrical Shells," Experimental Mechanics, Vol. 13, 1973, pp. 487-492.

${ }^{12}$ Bull, J. W., "Stresses Around Large Circular Holes in Uniform Circular Cylindrical Shells," Journal of Strain Analysis, Vol. 17, 1982, pp. 9-12.

${ }^{13}$ Zirka, A. I. and Chernopiskii, "Stress Concentration in an Axially Compressed Cylindrical Shell of Medium Thickness with an Elliptic Opening," International Applied Mechanics, Vol. 10, 2003, pp. 1466-1472.

${ }^{14}$ Liang, C., Hsu C. and Chen W., "Curvature Effect on Stress Concentrations Around Circular Hole in Opened Shallow Cylindrical Shell Under External Pressure," International Journal of Pressure Vessels and Piping, Vol. 75, 1998, pp. 749-763.

${ }^{15}$ Shnerenko, K. I. and Godzula, V. F., "Stress Distribution in a Composite Cylindrical Shell with a Large Circular Opening," International Applied Mechanics, Vol. 39, 2003, pp. 1323-1327.

${ }^{16}$ Hicks, R., "Stress Concentrations Around Holes in Plates and Shells," Proceedings of the Applied Mechanics Conference, New Castle Upon Tyne, 1964, pp. 3-12.

${ }^{17}$ Ebner, H. and Jung, O., 1972, "Stress Concentration Around Holes in Plates and Shells," Contributions to the Theory of Aircraft Structures, Delft University Press, Rotterdam.

${ }^{18} \mathrm{Li}$, Y. W., Elishakoff, I., and Starnes, J.H., Jr., "Axial Buckling of Composite Cylindrical Shells with Periodic Thickness Variation," Computers and Structures, Vol. 56, 1995, pp. 65-74.

${ }^{19}$ Sheinman, I. and Firer, M., "Buckling Analysis of Laminated Cylindrical Shells with Arbitrary Noncircular Cross Sections," AIAA Journal, Vol. 32, 1994, pp. 648-654.

${ }^{20}$ Hyer, M. W. and Wolford, G. F., "Progressive Failure Analysis of Internally Pressurized Noncircular Composite Cylinders," $43^{\text {rd }}$ AIAA/ASME/ASCE/AHS/ASC Structures, Structural Dynamics, and Materials Conference, Denver, Colorado, Paper No. 2002-1403, 2002.

${ }^{21}$ Hyer, M. W. and Wolford, G. F., "Damage Initiation and Progression in Internally Pressurized Noncircular Composite Cylinders," $44^{\text {rd }}$ AIAA/ASME/ASCE/AHS/ASC Structures, Structural 
Dynamics, and Materials Conference, Norfolk, Virginia, Paper No. 2003-1594, 2003.

${ }^{22}$ Lekhnitskii, S. G., Anisotropic Plates, Gordon and Breach Science Publishers, Inc., New York, 1968.

${ }^{23}$ Romano, F. and Kempner, J., "Stress and Displacement Analysis of a Simply Supported Noncircular Cylindrical Shells under Lateral Pressure," PIBAL Report No. 415, Polytechnic Institute of Brooklyn, New York, 1958.

${ }^{24}$ Shewchuk, J. R, "Triangle: Engineering a 2D Quality Mesh Generator and Delaunay Triangulator," First Workshop on Applied Computational Geometry, Philadelphia, Pennsylvania, pp. 124-133, 1996.

${ }^{25}$ Madenci, E. and Barut, A., "Influence of an Elliptical Cutout on Buckling Response of Composite Cylindrical Shells with Non-uniform Wall-Thickness and Non-Circular Cross-Section," $44^{\text {rd }}$ AIAA/ASME/ASCE/AHS/ASC Structures, Structural Dynamics, and Materials Conference, Norfolk, Virginia, Paper No. 2003-1929, 2003.

${ }^{26}$ Love, A. E. H., "The Small Free Vibrations and Deformation of a Thin Elastic Shell," Philosophical Transactions of the Royal Society of London, Vol. 179, A, 1888.

${ }^{27}$ Love, A. E. H., A Treatise on the Mathematical Theory of Elasticity, 4th ed., Dover Publications, New York, 1944.

${ }^{28}$ Timoshenko, S. and Woinowsky-Krieger, S., Theory of Plates and Shells, 2nd ed., McGraw-Hill Book Company, New York, 1959.

${ }^{29}$ Bushnell, D., "Computerized Analysis of Shells Governing Equations," Computers \& Structures, Vol. 18, No. 3, 1984, pp. 471-536.

${ }^{30}$ Reissner, E., "A New Derivation of the Equations for thr Deformation of elastic Shells," American Journal of Mathematics, Vol. 63, 1941, pp. 177-184.

${ }^{31}$ Kraus, H., Thin Elastic Shells - An Introduction to the Theoretical Foundations and the Analysis of Their Static and Dynamic Behavior, John Wiley and Sons, Inc., 1967.

${ }^{32}$ Naghdi, P. M., "Foundations of Elastic Shell Theory," Office of Naval Research, Technical Report No. 15, January 1962.

${ }^{33}$ Chaudhuri, R. A., Balaraman, K., and Kunukkasseril, V. X., "Arbitrarily Laminated, Anisotropic Cylindrical Shell Under Internal Pressure," AIAA Journal, Vol. 24, No. 11, 1986, pp. 1851-1858.

${ }^{34}$ Jones, R. M., Mechanics of Composite Materials, 2nd ed., Taylor \& Francis, Inc. Philadelphia, Pennsylvania, 1999.

${ }^{35}$ McFarland, D., Bert L. Smith, B. L. and Walter D. Bernhart, W. D., Analysis of Plates, Spartan Books, New York, 1972.

${ }^{36}$ Madenci, E. and Barut, A., "A Free-Formulation Based Flat Shell Element for Non-Linear Analysis of Thin Composite Structures," International Journal for
Numerical Methods in Engineering, Vol. 37, 1994, pp. 3825-3842.

${ }^{37}$ Madenci, E. and Barut, A., "Pre- and Postbuckling Response of Curved, Thin Composite Panels with Cutouts under Compression," International Journal for Numerical Methods in Engineering, Vol. 37, 1994, pp. 1499-1510.

${ }^{38}$ Madenci, E. and Barut, A., "Thermal Postbuckling Analysis of Cylindrically Curved Composite Laminates with a Hole," International Journal for Numerical Methods in Engineering, Vol. 37, 1994, pp. 2073-2091.

\section{Appendix A}

\section{Strain Energy of shell}

Based on classical laminated shell theory, the strain energy of the shell can be expressed as

$U=\frac{1}{2} \int_{A} \mathbf{s}^{T} \mathbf{e} d A$

in which $A$ is the planform area of the shell midsurface. Substituting the expressions for the resultant stress and strains, given in terms of the vector of unknown displacement coefficients, q, by Eqs. (35) and (37), leads to

$U(\mathbf{q})=\frac{1}{2} \int_{A} \mathbf{q}^{T}\left(\mathbf{L}^{T} \mathbf{C} \mathbf{L}\right) \mathbf{q} d A$

The matrix $\mathbf{L}$ involves the derivatives of the assumed, functional displacement representations, and $\mathbf{C}$ is the overall constitutive matrix defined by Eq. (16c). The expression for the strain energy is rewritten into the final form used herein as

$U(\mathbf{q})=\frac{1}{2} \mathbf{q}^{T} \mathbf{k}_{q q} \mathbf{q}$

where

$\mathbf{k}_{q q}=\int_{A}\left(\mathbf{L}^{T} \mathbf{C} \mathbf{L}\right) d A$

The evaluation of this area integral is performed numerically by employing basic quadrature techniques. In this analysis, the quadrature points are predetermined by employing standard triangulation of the entire domain as described by Shewchuk. ${ }^{24}$

\section{Strain energy of elastic restraints}

The strain energy of the elastic edge restraints (springs), $\Omega$, is expressed as 


$$
\begin{aligned}
\Omega= & \frac{1}{2} \sum_{\ell=1}^{2} \sum_{\alpha=n, s, t} \int_{\Gamma_{(\ell)}} S_{\alpha}\left(u_{\alpha}-u_{\alpha}^{*}\right)^{2} d \Gamma \\
& +\frac{1}{2} \sum_{\ell=1}^{2} \sum_{\alpha=n, s} \int_{\Gamma_{(\ell)}} J_{\alpha}\left(\vartheta_{\alpha}-\vartheta_{\alpha}^{*}\right)^{2} d \Gamma \\
& +\frac{1}{2} \sum_{\ell=1}^{2} \sum_{\alpha=n, s} \int_{\Gamma_{(\ell)}} s_{\alpha}\left(u_{\alpha}-\Delta_{\alpha}\right)^{2} d \Gamma+
\end{aligned}
$$

As depicted in Fig. 4, the boundary displacements $u_{n}, u_{s}$, and $u_{t}$ along the $\ell^{\text {th }}$ boundary segment are restrained by extensional springs with the stiffness values $S_{n}, S_{s}$, and $S_{t}$, respectively. Likewise, the boundary rotations $\vartheta_{n}$ and $\vartheta_{s}$ are restrained by rotational springs with the stiffness values $J_{n}$ and $J_{s}$, respectively.

In order to apply concentrated forces along the edge of a shell and introduce edge displacements that are similar to those introduced by a rigid end-ring or by the loading platens of a testing machine, additional springs are uses to simulate the load-introduction effects of a rigid end-ring. In particular, rigid-end-ring loads are introduced into the shell by using extensional springs in the directions normal and tangent to the boundary with corresponding stiffness values of $s_{n}$ and $s_{s}$, as shown in Fig. 3. By specifying relatively large values for the spring stiffnesses $s_{n}$ and $s_{s}$, the laminate edge behaves as if a rigid end-ring is attached that produces the uniform displacements $\Delta_{n}$ and $\Delta_{s}$. In contrast, a relatively small spring stiffness between the shell edge and the rigid end-ring eliminates the presence of a rigid end-ring.

The desired form of the elastic-restraint strain energy is obtained in terms of the unknown vector $\mathbf{q}$ by substituting expressions for the boundary displacements and rotations, given collectively by Eq. (29), into Eq. (42). This step yields

$$
\begin{aligned}
\Omega & =\frac{1}{2} \sum_{\ell=1}^{2} \sum_{\alpha=n, s, t}\left(\mathbf{q}^{T} \mathbf{S}_{\alpha \alpha}^{(\ell)} \mathbf{q}+\Omega_{u(\alpha)}^{(\ell)}{ }^{*}-2 \mathbf{q}^{T} \mathbf{f}_{\alpha}^{(\ell)^{*}}\right) \\
& +\frac{1}{2} \sum_{\ell=1}^{2} \sum_{\alpha=n, s}\left(\mathbf{q}^{T} \mathbf{J}_{\alpha \alpha}^{(\ell)} \mathbf{q}+\Omega_{\vartheta(\alpha)}^{(\ell)^{*}}-2 \mathbf{q}^{T} \mathbf{r}_{\alpha}^{(\ell)^{*}}\right) \\
& +\frac{1}{2} \sum_{\ell=1}^{2} \sum_{\alpha=n, s}\left(\mathbf{q}^{T} \mathbf{s}_{\alpha \alpha}^{(\ell)} \mathbf{q}+\int_{\Gamma_{(\ell)}} s_{\alpha} \Delta_{\alpha}^{2} d \Gamma-2 \mathbf{q}^{T} \mathbf{s}_{\alpha}^{(\ell)} \Delta_{\alpha}\right)
\end{aligned}
$$

where the matrices $\mathbf{S}_{\alpha \alpha}^{(\ell)}$ and $\mathbf{J}_{\alpha \alpha}^{(\ell)}$ represent the stiffness contribution of the extensional and rotational springs attached to the $\ell$ th segment of the boundary. These matrices are defined as

$$
\mathbf{S}_{\alpha \alpha}^{(\ell)}=\int_{\Gamma_{(\ell)}} S_{\alpha} \mathbf{u}_{\alpha} \mathbf{u}_{\alpha}^{T} d \Gamma \quad(\alpha=n, s, t)
$$

and
$\mathbf{J}_{\alpha \alpha}^{(\ell)}=\int_{\Gamma_{(\ell)}} J_{\alpha} \boldsymbol{\theta}_{\alpha} \boldsymbol{\theta}_{\alpha}^{T} d \Gamma \quad(\alpha=n, s)$

The matrix $\mathbf{s}_{\alpha \alpha}^{(\ell)}$, representing the stiffness of the springs attached to the rigid end-ring, is defined as

$\mathbf{s}_{\alpha \alpha}^{(\ell)}=\int_{\Gamma_{(\ell)}} s_{\alpha} \mathbf{u}_{\alpha} \mathbf{u}_{\alpha}^{T} d \Gamma \quad(\alpha=n, s)$

The load vectors, $\mathbf{f}_{\alpha}^{(\ell)^{*}}$ and $\mathbf{r}_{\alpha}^{(\ell)^{*}}$, are associated with the prescribed boundary displacements and rotations and are defined as

$$
\mathbf{f}_{\alpha}^{(\ell)^{*}}=\int_{\Gamma_{(\ell)}} S_{\alpha} u_{\alpha}^{*} \mathbf{u}_{\alpha} d \Gamma \quad(\alpha=n, s, t)
$$

and

$\mathbf{r}_{\alpha}^{(\ell)^{*}}=\int_{\Gamma_{(\ell)}} J_{\alpha} \vartheta_{\alpha}^{*} \boldsymbol{\theta}_{\alpha} d \Gamma \quad(\alpha=n, s)$

The vector, $\mathbf{s}_{\alpha}^{(\ell)}$, is associated with the unknown enddisplacements that correspond to a given concentrated load and is defined as

$\mathbf{s}_{\alpha}^{(\ell)}=\int_{\Gamma_{(\ell)}} s_{\alpha} \mathbf{u}_{\alpha} d \Gamma \quad(\alpha=n, s)$

The strain energies in the springs that arises from the known prescribed displacements $\left(u_{n}^{*}, u_{s}^{*}\right.$ and $\left.u_{t}^{*}\right)$ and rotations ( $\vartheta_{n}^{*}$ and $\vartheta_{s}^{*}$ ) are defined as

$\Omega_{u(\alpha)}^{(\ell)}{ }^{*}=\int_{\Gamma_{(\ell)}} S_{\alpha} u_{\alpha}^{* 2} d \Gamma \quad(\alpha=n, s, t)$

and

$\Omega_{\vartheta(\alpha)}^{(\ell)}{ }^{*}=\int_{\Gamma_{(\ell)}} J_{\alpha} \vartheta_{\alpha}^{* 2} d \Gamma \quad(\alpha=n, s)$

For convenience, the expression for the strain energy in the springs is recast in matrix form as

$$
\begin{aligned}
\Omega(\mathbf{q}, \Delta)= & \frac{1}{2} \mathbf{q}^{T} \mathbf{S}_{q q} \mathbf{q}+\frac{1}{2} \Delta^{T} \mathbf{s}_{\Delta \Delta} \Delta \\
& -\mathbf{q}^{T} \mathbf{s}_{q \Delta} \Delta-\mathbf{q}^{T} \mathbf{f}^{*}+\Omega^{*}
\end{aligned}
$$

in which the matrices, $\mathbf{S}_{q q}, \mathbf{s}_{\Delta \Delta}$ and $\mathbf{s}_{q \Delta}$ represent the stiffness of the springs associated with the deformation of the laminate, the end-displacements and their coupling, respectively. These matrices are defined by

$$
\begin{aligned}
& \mathbf{S}_{q q}=\sum_{\ell=1}^{2} \sum_{\alpha=n, s, t} \mathbf{S}_{\alpha \alpha}^{(\ell)}+\sum_{\ell=1}^{2} \sum_{\alpha=n, s} \mathbf{J}_{\alpha \alpha}^{(\ell)}+\sum_{\ell=1}^{2} \sum_{\alpha=n, s} \mathbf{s}_{\alpha \alpha}^{(\ell)} \\
& \mathbf{s}_{\Delta \Delta}=\operatorname{Diag}\left[s_{n}^{(1)}, s_{n}^{(2)}, s_{s}^{(1)}, s_{s}^{(2)}\right] \times 2 \pi R_{0} \\
& \mathbf{S}_{q \Delta}=\left[\begin{array}{llll}
\mathbf{s}_{n}^{(1)} & \mathbf{s}_{n}^{(2)} & \mathbf{s}_{s}^{(1)} & \mathbf{s}_{s}^{(2)}
\end{array}\right]
\end{aligned}
$$

The vector of unknown end-displacements, $\boldsymbol{\Delta}$, is defined by

$$
\Delta^{T}=\left\{\Delta_{n}^{(1)}, \Delta_{n}^{(2)}, \Delta_{s}^{(1)}, \Delta_{s}^{(2)}\right\}
$$

The load vectors arising from all prescribed boundary displacements and rotations, $\mathbf{f}^{*}$, is defined as 


$$
\mathbf{f}^{*}=\frac{1}{2} \sum_{\ell=1}^{2} \sum_{\alpha=n, s, t} \mathbf{f}_{\alpha}^{*(\ell)}+\frac{1}{2} \sum_{\ell=1}^{2} \sum_{\alpha=n, s} \mathbf{r}_{\alpha}^{*(\ell)}
$$

and the strain energy of all the springs due to prescribed displacements and rotations is

$$
\Omega^{*}=\frac{1}{2} \sum_{\ell=1}^{2} \sum_{\alpha=n, s, t} \Omega_{u(\alpha)}^{(\ell)}{ }^{*}+\frac{1}{2} \sum_{\ell=1}^{2} \sum_{\alpha=n, s} \Omega_{\vartheta(\alpha)}^{(\ell)}{ }^{*}
$$

\section{Potential of external loads}

The potential energy of the external tractions $\left(t_{n}^{*}, t_{s}^{*}\right.$ and $\left.t_{t}^{*}\right)$ and moments $\left(m_{n}^{*}\right.$ and $\left.m_{s}^{*}\right)$ acting along the $\ell^{\text {th }}$ boundary segment, and the concentrated loads $\left(P_{n}^{*}\right.$ and $\left.\mathrm{P}_{s}^{*}\right)$ acting on the rigid end rings, is given in terms of the corresponding boundary displacements and rotations by

$$
\begin{aligned}
V & =-\sum_{\ell=1}^{2} \sum_{\alpha=n, s, t} \int_{\Gamma_{(\ell)}} t_{\alpha}^{*} u_{\alpha} d \Gamma \\
& -\sum_{\ell=1}^{2} \sum_{\alpha=n, s} \int_{\Gamma_{(\ell)}} m_{\alpha}^{*} \vartheta_{\alpha} d \Gamma-\sum_{\ell=1}^{2} \sum_{\alpha=n, s} P_{\alpha}^{*} \Delta_{\alpha}
\end{aligned}
$$

Substituting the expressions for the boundary displacements and rotations, given in terms of the vector $\mathbf{q}$, and combining terms in Eq. (55) yields

$$
V(\mathbf{q}, \Delta)=-\mathbf{q}^{T} \mathbf{T}^{*}-\Delta^{T} \mathbf{P}^{*}
$$

where the vector $\Delta$, containing the uniform enddisplacements $\Delta_{n}$ and $\Delta_{s}$ of the $\ell^{\text {th }}$ boundary segment, is defined by

$$
\Delta^{\mathrm{T}}=\left\{\Delta_{n}^{(1)}, \Delta_{n}^{(2)}, \Delta_{s}^{(1)}, \Delta_{s}^{(2)}\right\}
$$

The load vectors, $\mathbf{T}^{*}$ and $\mathbf{P}^{*}$ are defined by

$$
\mathbf{T}^{* T}=\sum_{\ell=1}^{2} \sum_{\alpha=n, s, t} \int_{\Gamma_{(\ell)}} t_{\alpha}^{*} \mathbf{u}_{\alpha}^{T} d \Gamma+\sum_{\ell=1}^{2} \sum_{\alpha=n, s} \int_{\Gamma_{(\ell)}} \vartheta_{\alpha}^{*} \boldsymbol{\theta}_{\alpha}^{T} d \Gamma
$$

and

$$
\mathbf{P}^{* T}=\left\{P_{n}^{*(1)}, P_{n}^{*(2)}, P_{s}^{*(1)}, P_{s}^{*(2)}\right\}
$$

in which ${ }^{(\ell)} P_{\alpha}^{*}$, with $(\alpha=n, s)$, represents the membrane forces applied on the $\ell^{\text {th }}$ boundary segment through a rigid end-ring.

\section{$\underline{\text { Appendix B }}$}

\section{Rigid-body modes}

As given by Madenci and Barut ${ }^{24}$, the rigid-body displacements $\left(u_{R 1}, u_{R 2}\right.$ and $\left.u_{R 3}\right)$ of a cylindrical shell, defined with respect to the curvilinear coordinates, $\left(s_{1}, s_{2}, s_{3}\right)$, are expressed herein as

$$
\begin{aligned}
& u_{R 1}=\alpha_{1}-\alpha_{6} y+\alpha_{5} z \\
& u_{R 2}=\alpha_{2} \cos \theta-\alpha_{3} \sin \theta-\alpha_{4}(y \sin \theta+z \cos \theta) \\
& +\alpha_{5} x \sin \theta+\alpha_{6} x \cos \theta
\end{aligned}
$$

$$
\begin{aligned}
u_{R 3}=\alpha_{2} \sin \theta+\alpha_{3} \cos \theta & +\alpha_{4}(y \cos \theta-z \sin \theta) \\
& -\alpha_{5} x \cos \theta+\alpha_{6} x \sin \theta
\end{aligned}
$$

where $\theta$ denotes the angle between the radius of curvature at a point on the shell surface and $z$-axis as shown in Fig. 1.

\section{Global functions}

The global functions $\bar{u}_{i}$ that are used to capture the overall deformations away from the cutout are expressed in terms of a series expansion of orthogonal functions of the form

$\bar{u}_{i}\left(s_{1}, s_{2}\right)=\sum_{m=0}^{M} \sum_{n=0}^{m} c_{i(m n)} T_{m}\left(s_{1}\right) W_{n}\left(s_{2}\right)$

The symbols $c_{i(m n)}$ are the unknown real-valued coefficients, and $T_{m}\left(s_{1}\right)$ and $W_{n}\left(s_{2}\right)$ are defined as

$$
T_{m}\left(s_{1}\right)= \begin{cases}1 & m=0 \\ \zeta & m=1 \\ \sin \left[\frac{(m-1)}{2}(\zeta+1)\right] & m>1\end{cases}
$$

and

$W_{n}\left(s_{2}(\theta)\right)= \begin{cases}\cos (n \theta / 2) & n=0,2,4,6,8, \cdots \\ \sin ((n+1) \theta / 2) & n=1,3,5,7,9, \cdots\end{cases}$

in which $-1 \leq \zeta \leq 1$ and $s_{1}$ is related to $\zeta$ as $s_{1}=\zeta L / 2$, with $L$ being the length of the cylinder. Note that $W_{n}$ is periodical. These particular functions were chosen because they form a complete set of functions when used with Eq. (59). Hence, they are desirable for employing in energy based semi-analytic solution techniques such as the total potential energy principal that is used in this study.

\section{Local functions}

The local functions are expressed in terms of mapping functions that transform the contour of an elliptical cutout to a unit circle. These mapping functions are used permit the use of Laurent series expansions as local functions, which is desirable because Laurent series are analytic and uniformly convergent in domains with a circular hole. As a result, the use of mapping functions reduces the number of terms in the Laurent series significantly that are needed to adequately capture steep stress and strain gradients and local deformations near a cutout. In accordance with the principle of minimum potential energy, the local local functions are not required to satisfy the traction boundary conditions at the cutout boundary. Thus, the local functions, $\overline{\bar{u}}_{i}$, are expressed in the form of Laurent series, in terms of complex functions, as 


$$
\begin{aligned}
& \overline{\bar{u}}_{1}=2 \operatorname{Re}\left[\sum_{m=1}^{2} u_{m}^{(1)} \sum_{\substack{n=-N \\
n \neq 0}}^{N} \alpha_{n m} \Phi_{n m}^{*}\left(z_{\varepsilon m}\right)\right] H(\rho) \\
& \overline{\bar{u}}_{2}=2 \operatorname{Re}\left[\sum_{m=1}^{2} u_{m}^{(2)} \sum_{\substack{n=-N \\
n \neq 0}}^{N} \alpha_{n m} \Phi_{n m}^{*}\left(z_{\varepsilon m}\right)\right] H(\rho) \\
& \overline{\bar{u}}_{3}=2 \operatorname{Re}\left[\sum_{m=1}^{2} \sum_{\substack{n=-N \\
n \neq 0}}^{N} \beta_{n m} F_{n m}^{*}\left(z_{\kappa m}\right)\right] H(\rho)
\end{aligned}
$$

with

$$
\rho=\sqrt{x_{1}^{2}+x_{2}^{2}}
$$

where the parameter $N$ defines the extent of the complex series. In these series, $\alpha_{n m}$ and $\beta_{n m}$ are the unknown complex coefficients that appear in Eqs. (26)(28). The auxiliary function $H(\rho)$ that defines the domain of influence of the local functions is expressed in a polynomial form as

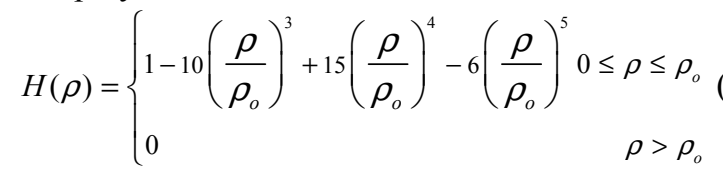

with

$$
H\left(\rho_{o}\right)=H^{\prime}\left(\rho_{o}\right)=H^{\prime \prime}\left(\rho_{o}\right)=0
$$

where the prime marks denotes differentiation with respect to the variable $\rho$ and the parameter $\rho_{o}$ denotes the radius of the region in which the local functions are effective. The purpose of chosing the auxiliary function is to prevent any possible linear dependency between the local and global functions and to restrict the influence of the local functions to a limited domain around the cutout.

The complex functions $u_{m}^{(1)}\left(z_{\varepsilon m}\right)$ and $u_{m}^{(2)}\left(z_{\varepsilon m}\right)$ that appear in Eqs. (61a) and (61b) are defined as

$u_{m}^{(1)}\left(z_{\varepsilon m}\right)=\cos \psi \quad p_{m}\left(z_{\varepsilon m}\right)-\sin \psi \quad q_{m}\left(z_{\varepsilon m}\right)$

$u_{m}^{(2)}\left(z_{\varepsilon m}\right)=\sin \psi \quad p_{m}\left(z_{\varepsilon m}\right)+\cos \psi \quad q_{m}\left(z_{\varepsilon m}\right)$

where the complex constants $p_{m}$ and $q_{m}$ are given by

$p_{m}=a_{11} \mu_{\varepsilon m}^{2}+a_{12}-a_{16} \mu_{\varepsilon m}$

$q_{m}=a_{12} \mu_{\varepsilon m}+a_{22} / \mu_{\varepsilon m}-a_{26}$

In Eqs. (65a) and (65b), the unknown complex constants, $\mu_{\varepsilon m}$, are the roots to the characteristic equation associated with membrane deformation, i.e.,

$$
\begin{aligned}
a_{11} \mu_{\varepsilon m}^{4}-2 a_{16} \mu_{\varepsilon m}^{3}+\left(2 a_{26}+a_{66}\right) \mu_{\varepsilon m}^{2} & \\
& -2 a_{26} \mu_{\varepsilon m}+a_{22}=0
\end{aligned}
$$

in which the coefficients $a_{i j}$ are the coefficients of the flexibility matrix $\mathbf{a}$, which is the inverse of the stiffness matrix A defined by Eq. (13a). Both the flexibility and the stiffness matrices, $\mathbf{a}$ and $\mathbf{A}$, are measured with respect to the local coordinate $\operatorname{system}\left(x_{1}, x_{2}\right)$. The angle, $\psi$ represents the orientation of the local coordinate system with respect to the global coordinate system, $\left(s_{1}, s_{2}\right)$.

The complex potential function, $\Phi_{n m}^{*}\left(z_{\varepsilon_{m}}\right)$, appearing in Eqs. (61a) and (61b) is defined as $\Phi_{n m}^{*}\left(z_{\varepsilon m}\right)=\xi_{\varepsilon m}^{n}$

in which the mapping functions, $\xi_{\varepsilon m}$, map a cutout onto a unit circle. The mapping functions for an elliptical cutout, introduced by Lekhnitskii ${ }^{22}$, are given by

$$
\xi_{\varepsilon m}=\frac{z_{\varepsilon m} \pm \sqrt{z_{\varepsilon m}^{2}-a^{2}-\mu_{\varepsilon m}^{2} b^{2}}}{a-i \mu_{\varepsilon m}^{2} b} \quad(m=1,2)
$$

where $z_{\varepsilon m}=x_{1}+\mu_{\varepsilon m} x_{2}, a$ and $b$ are the major and minor axes of the elliptical cutout, and $i=\sqrt{-1}$. The sign of the square-root term is chosen so that $\left|\xi_{\varepsilon m}\right| \geq 1$ (i.e., the mapped point is guaranteed to be on or outside the unit circle).

Inverting the mapping function provides $\omega_{\varepsilon m}\left(\xi_{\varepsilon m}\right)$ as

$$
z_{\varepsilon m}=\omega_{\varepsilon m}\left(\xi_{\varepsilon m}\right)=r_{\varepsilon m} \xi_{\varepsilon m}-\frac{s_{\varepsilon m}}{\xi_{\varepsilon m}}
$$

in which

$r_{\varepsilon m}=\frac{1}{2}\left(a-i \mu_{\varepsilon m} b\right), \quad s_{\varepsilon m}=\frac{1}{2}\left(a+i \mu_{\varepsilon m} b\right)$

The unknown complex constants $\mu_{\varepsilon 1}$ and $\mu_{\varepsilon 2}$, and their complex conjugates, i.e., $\mu_{\varepsilon 3}=\bar{\mu}_{\varepsilon 1}$ and $\mu_{\varepsilon 4}=\bar{\mu}_{\varepsilon 2}$, are the roots obtained from the characteristic equation associated with membrane deformation

The complex potential functions, $F_{n m}^{*}\left(z_{\kappa m}\right)$ in E. (61c) are defined as

$$
F_{n m}^{*}\left(z_{\kappa m}\right)= \begin{cases}\frac{r_{\kappa m}}{n+1} \xi_{\kappa m}^{n}-\frac{s_{K m}}{n-1} \xi_{\kappa m}^{n-2}, & |n|>1 \\ \frac{r_{\kappa m}}{2} \xi_{\kappa m}^{2}-s_{\kappa m} \ln \xi_{\kappa m}, & n=1 \\ r_{\kappa m} \ln \xi_{\kappa m}+\frac{s_{\kappa m}}{2} \xi_{\kappa m}^{-2}, & n=-1\end{cases}
$$

in which the expressions for the mapping function $\xi_{k m}$ and the constants $r_{K m}$ and $s_{K m}$ have the same form as the corresponding expressions for $\xi_{\varepsilon m}, r_{\varepsilon m}$, and $s_{\varepsilon m}$ given by Eqs. (68) - $(70 \mathrm{a}, \mathrm{b})$, except that the subscript $\varepsilon$ is replaced by $\kappa$.

The complex variables $z_{\kappa m}$ are defined by

$$
z_{\kappa m}=x_{1}+\mu_{\kappa m} x_{2}
$$


in which the unknown complex constants $\mu_{\kappa 1}$ and $\mu_{\kappa 2}$ and their conjugates, i.e., $\mu_{\kappa 3}=\bar{\mu}_{\kappa 1}$ and $\mu_{\kappa 4}=\bar{\mu}_{\kappa 2}$, are the roots obtained from the characteristic equation associated with the bending equilibrium equation

$$
\begin{aligned}
D_{22} \mu_{\kappa m}^{4}+4 D_{26} \mu_{\kappa m}^{3}+\left(2 D_{12}\right. & \left.+4 D_{66}\right) \mu_{\kappa m}^{2} \\
& +4 D_{16} \mu_{\kappa m}+D_{11}=0
\end{aligned}
$$

where $D_{i j}$ are the components of the bending stiffness matrix D (see Eq. (13b), which is defined with respect to the local coordinate system, $\left(x_{1}, x_{2}\right)$.

It is important to note that the local functions in Eq. (61) satisfy the in-plane and bending equilibrium equations of a homogeneous, flat laminate of uniform thickness, not a cylindrical shell. Therefore, the roots to the characteristic equations, Eqs. (66) and (73) serve as approximation to their exact values which are not mathematically tractable. Because the solution procedure is based on the principle of minimum potential, their exact values are not necessarily required. However, they capture the stress concentration and local deformation near the cutout in cylindrical shells because these functions possess the inherent solution characteristics. They satify the equilibrium equations exactly as the radius of curvature approaches inifinity and are uniformly convergent in a doubly connected region.

In the displacement representations defined by Eqs. (26a) and (26b), the vectors, $\boldsymbol{\alpha}_{R}, \mathbf{c}_{i}, \boldsymbol{\alpha}$, and $\boldsymbol{\beta}$ are defined as

$$
\begin{gathered}
\boldsymbol{\alpha}_{R}{ }^{T}=\left\{\alpha_{R 1}, \alpha_{R 2}, \alpha_{R 3}, \alpha_{R 4}, \alpha_{R 5}, \alpha_{R 6}\right\} \\
\mathbf{c}_{i}^{T}=\left\{c_{i(00)}, c_{i(10)}, c_{i(01)}, \ldots, c_{i(M 0)}\right. \\
\left., c_{i((M-1) 1)}, \ldots, c_{i(1(M-1))}, c_{i(0 M)}\right\} \\
\boldsymbol{\alpha}^{T}=\left\{\boldsymbol{\alpha}_{-N}^{T}, \boldsymbol{\alpha}_{-N+1}^{T}, \ldots, \boldsymbol{\alpha}_{-1}^{T}, \boldsymbol{\alpha}_{1}^{T}, \ldots, \boldsymbol{\alpha}_{N-1}^{T}, \boldsymbol{\alpha}_{N}^{T}\right\}
\end{gathered}
$$

in which

$$
\boldsymbol{\alpha}_{n}^{T}=\left\{\boldsymbol{\alpha}_{n 1}^{T}, \boldsymbol{\alpha}_{n 2}^{T}\right\}
$$

with

$$
\boldsymbol{\alpha}_{n j}^{T}=\left\{\operatorname{Re}\left[\alpha_{n j}\right], \quad \operatorname{Im}\left[\alpha_{n j}\right]\right\}
$$

and

$\boldsymbol{\beta}^{T}=\left\{\boldsymbol{\beta}_{-N}^{T}, \boldsymbol{\beta}_{-N+1}^{T}, \ldots, \boldsymbol{\beta}_{-1}^{T}, \boldsymbol{\beta}_{1}^{T}, \ldots, \boldsymbol{\beta}_{N-1}^{T}, \boldsymbol{\beta}_{N}^{T}\right\}$

in which

$$
\boldsymbol{\beta}_{n}^{T}=\left\{\boldsymbol{\beta}_{n 1}^{T}, \boldsymbol{\beta}_{n 2}^{T}\right\}
$$

with

$$
\boldsymbol{\beta}_{n j}^{T}=\left\{\operatorname{Re}\left[\beta_{n j}^{T}\right], \quad \operatorname{Im}\left[\beta_{n j}^{T}\right]\right\}
$$

The vector functions, $\mathbf{V}_{i}$, with $i=1,2,3$ associated with the unknown generalized coordinates $\mathbf{q}$ appearing in Eq. (27) are defined as

$$
\begin{array}{r}
\mathbf{V}_{1}^{T}=\left\{\mathbf{V}_{R 1}^{T}, \overline{\mathbf{V}}_{1}^{T}, \overline{\mathbf{0}}^{T}, \overline{\mathbf{0}}^{T}, \overline{\overline{\mathbf{V}}}_{1}^{T}, \overline{\overline{\mathbf{0}}}^{T}\right\} \\
\mathbf{V}_{2}^{T}=\left\{\mathbf{V}_{R 2}^{T}, \overline{\mathbf{0}}^{T}, \overline{\mathbf{V}}_{2}^{T}, \overline{\mathbf{0}}^{T}, \overline{\overline{\mathbf{V}}}_{2}^{T}, \overline{\overline{\mathbf{0}}}^{T}\right\} \\
\mathbf{V}_{3}^{T}=\left\{\mathbf{V}_{R 3}^{T}, \overline{\mathbf{0}}^{T}, \overline{\mathbf{0}}^{T}, \overline{\mathbf{V}}_{3}^{T}, \overline{\overline{\mathbf{0}}}^{T}, \overline{\overline{\mathbf{V}}}_{3}^{T}\right\}
\end{array}
$$

where

$\overline{\mathbf{0}}^{T}=\{0,0,0, . ., 0\}$ of order $[(\mathrm{M}+1)(\mathrm{M}+2) / 2]$

$\overline{\overline{\mathbf{0}}}^{T}=\{0,0, . ., 0\}$ of order $8 N$

The vectors associated with rigid body motion are

$\mathbf{V}_{R 1}^{T}=\{1,0,0,0, z,-y\}$

$$
\begin{array}{r}
\mathbf{V}_{R 2}^{T}=\left\{0, \frac{d y}{d s_{2}}, \frac{d z}{d s_{2}},\left(y \frac{d z}{d s_{2}}-z \frac{d y}{d s_{2}}\right)\right. \\
\left.,-x \frac{d z}{d s_{2}},-x \frac{d y}{d s_{2}}\right\}
\end{array}
$$

$$
\begin{array}{r}
\mathbf{V}_{R 3}^{T}=\left\{0,-\frac{d z}{d s_{2}}, \frac{d y}{d s_{2}},\left(y \frac{d y}{d s_{2}}+z \frac{d z}{d s_{2}}\right)\right. \\
\left.,-x \frac{d y}{d s_{2}},-x \frac{d z}{d s_{2}}\right\}
\end{array}
$$

Similarly, the vectors associated with the global functions are

$$
\begin{aligned}
\overline{\mathbf{V}}_{i}^{T}=\{ & T_{0}\left(s_{1}\right) W_{0}\left(s_{2}\right), T_{1}\left(s_{1}\right) W_{0}\left(s_{2}\right), \\
& T_{0}\left(s_{1}\right) W_{1}\left(s_{2}\right), T_{2}\left(s_{1}\right) W_{0}\left(s_{2}\right), \\
& T_{1}\left(s_{1}\right) W_{1}\left(s_{2}\right), T_{0}\left(s_{1}\right) W_{2}\left(s_{2}\right), . . \\
& \ldots, T_{M}\left(s_{1}\right) W_{0}\left(s_{2}\right), T_{M-1}\left(s_{1}\right) W_{1}\left(s_{2}\right), . . \\
& \left.\ldots, T_{1}\left(s_{1}\right) W_{M-1}\left(s_{2}\right), T_{0}\left(s_{1}\right) W_{M}\left(s_{2}\right)\right\}
\end{aligned}
$$

in which the expressions for $T_{i}\left(s_{1}\right)$ and $W_{i}\left(s_{2}\right)$ are given by Eq. (60), and

$$
\begin{aligned}
\overline{\overline{\mathbf{V}}}_{i}^{T}=\left\{\overline{\overline{\mathbf{V}}}_{i(-N)}^{T}, \overline{\overline{\mathbf{V}}}_{i(-N+1)}^{T}, \ldots, \overline{\overline{\mathbf{V}}}_{i(-1)}^{T}\right. \\
\left., \overline{\overline{\mathbf{V}}}_{i(1)}^{T}, \ldots, \overline{\overline{\mathbf{V}}}_{i(N-1)}^{T}, \overline{\overline{\mathbf{V}}}_{i(N)}^{T}\right\}
\end{aligned}
$$

with

$\overline{\overline{\mathbf{V}}}_{i(n)}^{T}=\left\{\overline{\overline{\mathbf{V}}}_{i(n 1)}^{T}, \overline{\overline{\mathbf{V}}}_{i(n 2)}^{T}\right\}$

in which

$\overline{\overline{\mathbf{V}}}_{i(n j)}^{T}=\left\{2 \operatorname{Re}\left[u_{j}^{(i)} \Phi_{n j}^{*}\right],-2 \operatorname{Im}\left[u_{j}^{(i)} \Phi_{n j}^{*}\right]\right\}$

where $i, j=1,2$ and

$$
\begin{aligned}
\overline{\overline{\mathbf{V}}}_{3}^{T}=\left\{\overline{\overline{\mathbf{V}}}_{3(-N)}{ }^{T}, \overline{\overline{\mathbf{V}}}_{3(-N+1)}^{T}, \ldots, \overline{\overline{\mathbf{V}}}_{3(-1)}^{T}\right. \\
\left., \overline{\overline{\mathbf{V}}}_{3(1)}^{T}, \ldots, \overline{\overline{\mathbf{V}}}_{3(N-1)}^{T}, \overline{\overline{\mathbf{V}}}_{3(N)}^{T}\right\}
\end{aligned}
$$

with

$\overline{\overline{\mathbf{V}}}_{3(n)}^{T}=\left\{\overline{\overline{\mathbf{V}}}_{3(n 1)}^{T}, \overline{\overline{\mathbf{V}}}_{3(n 2)}^{T}\right\}$

in which 


$$
\overline{\overline{\mathbf{V}}}_{3(n j)}^{T}=\left\{2 \operatorname{Re}\left[F_{n j}^{*}\right],-2 \operatorname{Im}\left[F_{n j}^{*}\right]\right\},(j=1,2)
$$

\section{Appendix C}

\section{Constraint Equations}

The unknown vector of Lagrange multipliers, $\lambda$ and the known coefficient matrix, G, in Eq. (19) are defined by

$$
\lambda^{T}=\left\{\lambda_{R R B(1)}, \lambda_{R R B(2)}, \lambda_{S V(r)}, \lambda_{S V(s)}, \lambda_{R B(1)}, \ldots, \lambda_{R B(6)}\right\}
$$

and

$$
\mathbf{G}=\left[\begin{array}{cccccc}
\mathbf{0}_{R}^{T} & \mathbf{g}_{R R B(1)}^{T} & \overline{\mathbf{0}}^{T} & \overline{\mathbf{0}}^{T} & \overline{\mathbf{0}}^{T} & \overline{\overline{\mathbf{0}}}^{T} \\
\mathbf{0}_{R}^{T} & \overline{\mathbf{0}}^{T} & \mathbf{g}_{R R B(2)}^{T} & \overline{\mathbf{0}}^{T} & \overline{\overline{\mathbf{0}}}^{T} & \overline{\overline{\mathbf{0}}}^{T} \\
\mathbf{0}_{R}^{T} & \overline{\mathbf{0}}^{T} & \overline{\mathbf{0}}^{T} & \overline{\mathbf{0}}^{T} & \overline{\overline{\mathbf{0}}}^{T} & \mathbf{g}_{S V(s)}^{T} \\
\mathbf{0}_{R}^{T} & \mathbf{0}_{L}^{T} & \overline{\mathbf{0}}^{T} & \overline{\mathbf{0}}^{T} & \overline{\mathbf{0}}^{T} & \mathbf{g}_{S V(r)}^{T} \\
\mathbf{g}_{R B(1)}^{T} & \overline{\mathbf{0}}^{T} & \overline{\mathbf{0}}^{T} & \overline{\mathbf{0}}^{T} & \overline{\overline{\mathbf{0}}}^{T} & \overline{\overline{\mathbf{0}}}^{T} \\
\mathbf{g}_{R B(2)}^{T} & \overline{\mathbf{0}}^{T} & \overline{\mathbf{0}}^{T} & \overline{\mathbf{0}}^{T} & \overline{\overline{\mathbf{0}}}^{T} & \overline{\overline{\mathbf{0}}}^{T} \\
\mathbf{g}_{R B(3)}^{T} & \overline{\mathbf{0}}^{T} & \overline{\mathbf{0}}^{T} & \overline{\mathbf{0}}^{T} & \overline{\mathbf{0}}^{T} & \overline{\overline{\mathbf{0}}}^{T} \\
\mathbf{g}_{R B(4)}^{T} & \overline{\mathbf{0}}^{T} & \overline{\mathbf{0}}^{T} & \overline{\mathbf{0}}^{T} & \overline{\overline{\mathbf{0}}}^{T} & \overline{\overline{\mathbf{0}}}^{T} \\
\mathbf{g}_{R B(5)}^{T} & \overline{\mathbf{0}}^{T} & \overline{\mathbf{0}}^{T} & \overline{\mathbf{0}}^{T} & \overline{\overline{\mathbf{0}}}^{T} & \overline{\overline{\mathbf{0}}}^{T} \\
\mathbf{g}_{R B(6)}^{T} & \overline{\mathbf{0}}^{T} & \overline{\mathbf{0}}^{T} & \overline{\mathbf{0}}^{T} & \overline{\mathbf{0}}^{T} & \overline{\mathbf{0}}^{T}
\end{array}\right]
$$

in which the constant coefficient vectors, $\mathbf{g}_{R R B(1)}$ and $\mathbf{g}_{R R B(2)}$ are associated with redundant rigidbody modes, $\mathbf{g}_{S V(r)}$ and $\mathbf{g}_{S V(s)}$ with single valuedness of the radial displacement component, and $\mathbf{g}_{R B(j)}$ with the rigid-body modes introduced by the global functions defined in Eq. (59). The rigid-body modes must be eliminated in the absence of a sufficient number of specified kinematic boundary conditions. These terms, as well as, the vectors with zeros are defined in the following subsections.

\section{Redundant rigid-body modes}

The coefficients, $c_{1(00)}$ and $c_{2(00)}$ in Eq. (59) for the global displacement functions produce additional rigidbody translation in the $s_{1}$ direction and rigid body rotation about the $s_{1}$ axis, respectively. Because these rigid-body modes are already represented by $\alpha_{R 1}$ and $\alpha_{R 6}$ in Eq. (58), the redundant rigid-body motion arising from the presence of $c_{1(00)}$ and $c_{2(00)}$, must be eliminated in order to obtain a unique representation of the displacements. These redundant rigid-body modes are eliminated by using the constraint conditions

$$
\begin{aligned}
& \lambda_{R R B(1)} c_{1(00)}=0 \\
& \lambda_{R R B(2)} c_{2(00)}=0
\end{aligned}
$$

in which the unknown Lagrange multipliers are denoted by $\lambda_{R R B(1)}$ and $\lambda_{R R B(2)}$. In terms of the vector of unknowns, $\mathbf{q}$, these constraints are rewritten in vector form as

$$
\begin{gathered}
\lambda_{R B(1)}\left\{\mathbf{0}_{R}^{T}, \mathbf{g}_{R R B}^{T}, \overline{\mathbf{0}}^{T}, \overline{\mathbf{0}}^{T}, \overline{\overline{\mathbf{0}}}^{T}, \overline{\overline{\mathbf{0}}}^{T}\right\}\left\{\begin{array}{l}
\boldsymbol{\alpha}_{R} \\
\mathbf{c}_{\mathbf{1}} \\
\mathbf{c}_{2} \\
\mathbf{c}_{3} \\
\boldsymbol{\alpha} \\
\boldsymbol{\beta}
\end{array}\right\}=0 \\
\lambda_{R B(2)}\left\{\mathbf{0}_{R}^{T}, \overline{\mathbf{0}}^{T}, \mathbf{g}_{R R B}^{T}, \overline{\mathbf{0}}^{T}, \overline{\overline{\mathbf{0}}}^{T}, \overline{\overline{\mathbf{0}}}^{T}\right\}\left\{\begin{array}{l}
\boldsymbol{\alpha}_{R} \\
\mathbf{c}_{\mathbf{1}} \\
\mathbf{c}_{2} \\
\mathbf{c}_{3} \\
\boldsymbol{\alpha} \\
\boldsymbol{\beta}
\end{array}\right\}=0
\end{gathered}
$$

in which the vectors of zeros, $\mathbf{0}_{R}^{T}, \overline{\mathbf{0}}_{R}^{T}$ and $\overline{\overline{\mathbf{0}}}_{R}^{T}$ are defined as

$\mathbf{0}_{R}^{T}=\{0,0,0,0,0,0\}$ (92a)

$\overline{\mathbf{0}}^{T}=\{0,0,0, \ldots, 0\}$ of order $[(\mathrm{M}+1)(\mathrm{M}+2) / 2]$

$\overline{\overline{\mathbf{0}}}^{T}=\{0,0,0, . ., 0\}$ of order $8 N(92 \mathrm{c})$

The constant coefficient vector, $\mathbf{g}_{R R B}$ is defined as

$\mathbf{g}_{R R B}^{T}=\{1,0,0, . ., 0\}$ of order $[(\mathrm{M}+1)(\mathrm{M}+2) / 2]$

\section{Single-valuedness of the radial displacement component}

The multi-valuedness of the normal displacement component that arises from the presence of logarithmic terms in the local expression for the radial displacement component in Eq. (61c) must be rendered single valued in order to obtain a unique solution. The logarithmic terms associated with complex constants, $\beta_{-1 m}$ and $\beta_{1 m}$, with $(m=1,2)$, in Eq. (71) result in two real constants. Representing the complex variable of the Laurent series, $\xi_{m}=\rho_{m} e^{i \theta_{m}}$, the single-valuedness requirement is enforced as

$u_{z}\left(\xi_{m}=\rho_{m} e^{i \theta_{m}}\right)-u_{z}\left(\xi_{m}=\rho_{m} e^{i\left(\theta_{m}+2 \pi\right)}\right)=0$ Associated with the complex constants, $\beta_{-1 m}$ and $\beta_{1 m}$ with $(m=1,2)$, in Eq. (71), this condition yields

$$
\begin{aligned}
2 \operatorname{Re}\left\{\sum_{m=1}^{2}\right. & \left\{F_{-1 m}^{*}\left(\xi_{m}=\rho_{m} e^{i \theta_{m}}\right)\right. \\
& \left.\left.-F_{-1 m}^{*}\left(\xi_{m}=\rho_{m} e^{i\left(\theta_{m}+2 \pi\right)}\right)\right\} \beta_{-1 m}\right\}=0
\end{aligned}
$$

or 


$$
\operatorname{Im} \sum_{m=1}^{2}\left[r_{m} \beta_{-1 m}\right]=0
$$

and

$$
\begin{aligned}
2 \operatorname{Re}\left\{\sum_{m=1}^{2}\right. & \left\{F_{1 m}^{*}\left(\xi_{m}=\rho_{m} e^{i \theta_{m}}\right)\right. \\
& \left.\left.-F_{1 m}^{*}\left(\xi_{m}=\rho_{m} e^{i\left(\theta_{m}+2 \pi\right)}\right)\right\} \beta_{1 m}\right\}=0
\end{aligned}
$$

or

$$
\operatorname{Im} \sum_{m=1}^{2}\left[s_{\kappa m} \beta_{1 m}\right]=0
$$

In order to ensure single-valuedness, these constraints are enforced as

$$
\lambda_{S V(r)} \operatorname{Im}\left[\sum_{m=1}^{2}\left[r_{\kappa m} \beta_{-1 m}\right]\right]=0
$$

and

$$
\lambda_{S V(s)} \operatorname{Im}\left[\sum_{m=1}^{2}\left[s_{\kappa m} \beta_{1 m}\right]\right]=0
$$

in which the unknown Lagrange multipliers are denoted by $\lambda_{S V(r)}$ and $\lambda_{S V(s)}$.

In terms of the vector of unknowns, $\mathbf{q}$, these constraint conditions can be recast in matrix form as

$\lambda_{S V(r)}\left\{\mathbf{0}_{R}^{T}, \overline{\mathbf{0}}^{T}, \overline{\mathbf{0}}^{T}, \overline{\mathbf{0}}^{T}, \overline{\overline{\mathbf{0}}}^{T}, \mathbf{g}_{S V(r)}^{T}\right\}$
$\left\{\begin{array}{l}\boldsymbol{\alpha}_{R} \\ \mathbf{c}_{\mathbf{1}} \\ \mathbf{c}_{2} \\ \mathbf{c}_{3} \\ \boldsymbol{\alpha} \\ \boldsymbol{\beta}\end{array}\right\}=0$
$\lambda_{S V(s)}\left\{\mathbf{0}_{R}^{T}, \overline{\mathbf{0}}^{T}, \overline{\mathbf{0}}^{T}, \overline{\mathbf{0}}^{T}, \overline{\overline{\mathbf{0}}}^{T}, \mathbf{g}_{S V(s)}^{T}\right\}$
$\left\{\begin{array}{l}\boldsymbol{\alpha}_{R} \\ \mathbf{c}_{1} \\ \mathbf{c}_{2} \\ \mathbf{c}_{3} \\ \boldsymbol{\alpha} \\ \boldsymbol{\beta}\end{array}\right\}=0$

where the constant coefficient vectors $\mathbf{g}_{S V(r)}$ and $\mathbf{g}_{S V(s)}$ are given by

$$
\begin{array}{r}
\mathbf{g}_{S V(j)}^{T}=\left\{\mathbf{g}_{S V(j)(-N)}^{T}, \mathbf{g}_{S V(j)(-N+1)}^{T}, \ldots, \mathbf{g}_{S V(j)(-1)}^{T}\right. \\
\left., \mathbf{g}_{S V(j)(1)}^{T}, \ldots, \mathbf{g}_{S V(j)(N-1)}^{T}, \mathbf{g}_{S V(j)(N)}^{T}\right\}
\end{array}
$$

in which

$$
\begin{aligned}
& \mathbf{g}_{S V(r)(1)}^{T}=\left\{\operatorname{Im}\left[r_{1}\right], \operatorname{Re}\left[r_{1}\right], \operatorname{Im}\left[r_{2}\right], \operatorname{Re}\left[r_{2}\right]\right\} \\
& \mathbf{g}_{S V(r)(n)}^{T}=\{0,0,0,0\} \quad \text { if } \quad n \neq 1
\end{aligned}
$$

and

$\mathbf{g}_{S V(s)(1)}^{T}=\left\{\operatorname{Im}\left[s_{1}\right], \operatorname{Re}\left[s_{1}\right], \operatorname{Im}\left[s_{2}\right], \operatorname{Re}\left[s_{2}\right]\right\}$

$\mathbf{g}_{S V(s)(n)}^{T}=\{0,0,0,0\} \quad$ if $\quad n \neq 1$

with $-N \leq n \leq N$.

\section{Rigid-body modes}

In the absence of kinematic boundary conditions, the rigid-body modes of the displacement field are eliminated by enforcing the constraint conditions in the form

$\lambda_{R B(j)} \alpha_{R(j)}=0$

where $j=1,2,3,4,5,6$, and the unknown Lagrange multipliers are denoted by $\lambda_{R B(j)}$. In terms of the vector of unknowns, $\mathbf{q}$, these constraints are rewritten in vector form as

$\lambda_{R B(j)}\left\{\mathbf{g}_{R B(j)}^{T}, \overline{\mathbf{0}}^{T}, \overline{\mathbf{0}}^{T}, \overline{\mathbf{0}}^{T}, \overline{\mathbf{0}}^{T}, \overline{\mathbf{0}}^{T}\right\}\left\{\begin{array}{c}\boldsymbol{\alpha}_{R} \\ \mathbf{c}_{1} \\ \mathbf{c}_{2} \\ \mathbf{c}_{3} \\ \boldsymbol{\alpha} \\ \boldsymbol{\beta}\end{array}\right\}=0$

where the constant coefficient vectors, $\mathbf{g}_{R B(j)}$ are defined as

$\mathbf{g}_{R B(j)}^{T}=\left\{\delta_{1 j}, \delta_{2 j}, \delta_{3 j}, \delta_{4 j}, \delta_{5 j}, \delta_{6 j}\right\}$

in which $\delta_{i j}$ is the Kronecker delta. 